\title{
General Synthesis of Tapered Matching Sections for Single Mode Operation Using the Coupled-Mode Theory
}

\author{
Jon M. Percaz, Israel Arnedo, Iván Arregui, Fernando Teberio, Petronilo Martin-Iglesias, \\ Miguel A. G. Laso, and Txema Lopetegi
}

\begin{abstract}
In this paper, a novel and general method to synthetize microwave waveguide tapers intended for single mode operation is proposed. The technique is based on the use of an exact series solution of the inverse scattering synthesis problem. An additional strategy necessary for dealing with waveguides where the propagation constant varies with the position is included. The coupled-mode theory is employed to model the electromagnetic behavior of the taper with the inherent mismatch caused by the connection of the waveguides with different cross-sections. The novel method allows us to synthesize the (classical) transmission line taper functions of Klopfenstein and Hecken, making them suitable for general waveguide tapers with single mode operation. Additionally, a new type of taper functions, also suitable for general waveguide tapers, is presented. The novel functions are obtained by partially employing the frequency response of multisection transformers, resulting in fully smooth tapers that can offer shorter lengths than the classical proposals. The taper synthesis procedure is demonstrated in rectangular waveguide technology, by requiring realistic and challenging specifications for different cases with different waveguide cross-sections to be matched: height mismatch, width mismatch, and simultaneous height and width mismatch. Several prototypes of Klopfenstein, Hecken and novel function tapers have been fabricated in an aluminum alloy by means of an Additive Manufacturing technique (Direct Metal Laser Sintering). The simulation and measurement results obtained for the rectangular waveguide taper prototypes confirm the accuracy of the novel synthesis technique proposed.
\end{abstract}

Index Terms - Coupled-mode Theory, Hecken, Inverse Scattering, Klopfenstein, Microwave Tapers, Tapered Matching Sections, Waveguide.

\section{INTRODUCTION}

$\mathrm{T}$ APERED matching sections, or simply tapers, for microwave transmission lines and waveguides are a mature research topic that can be traced back almost a century [1]-[3]. They can be defined as a smooth intermediate structure that allows us to connect two transmission lines or waveguides of different characteristic impedances or cross-sections, with a profile that varies continuously in a smooth fashion from one transmission line or waveguide to the other. The taper is designed to minimize the excitation of unwanted modes (including reflection), keeping the excitation level under a given design value. Following that broad definition it is easy to understand the importance of tapers, since they solve a common problem in numerous different microwave devices, technologies and systems.

Many different techniques for the design of tapers have been developed using the transmission line theory [4], [5]. Linear, hyperbolic, parabolic and exponential functions, among others, have been proposed. However, two sophisticated solutions must be highlighted. The first one was proposed by Klopfenstein [6] and later on completed by other authors [7]-[9], and it is also known as the Dolph-Chebyshev function. It achieves the optimum taper in the sense that its reflection level is below the maximum specified for the frequencies above the minimum required, with a tapering function of minimum length. However, the taper always exhibits discontinuities (steps) at its extremes that are inherent to the function. These critical steps can result in manufacturability problems and in the excitation of higher order modes that can be troublesome for certain applications. The second solution was proposed by Hecken [10] and it is also known as the modified Dolph-Chebyshev function. It is considered as a near-optimum taper and achieves a performance close to the Klopfenstein optimum proposal, employing a fully smooth tapering function with no discontinuities, but with a slightly larger length. Anyway, both Klopfenstein and Hecken tapers were developed for ideal transmission lines, in terms of the characteristic impedance parameter.

Very interesting proposals have been done to extend the theory of transmission line tapers to non-ideal transmission lines, synthesizing the taper in terms of the characteristic impedance but taking into account dispersion. Implementations in microstrip technology have been proposed [11], [12] and even tapers in finline have been carefully designed. For the finline case, the synthesis has been done in terms of the wave impedance [11] or using the mode coupling coefficient [13], [14] by introducing several approximations valid for that technology.

Tapers have been also widely studied and employed in microwave waveguide technologies, especially in rectangular and circular waveguides [15], [16]. Initially, simple tapers with linear profiles were proposed for circular (conical taper) [17] and rectangular waveguides (pyramidal taper) [18], [19]. The
Manuscript received Jan. 31, 2018. This work was supported by the MINECO (Spain) under project TEC2017-85529-C3-2-R (AEI, FEDER-EU). This paper is an expanded version from the IEEE Latin America Microwave Conference 2018, Arequipa, Peru, Dec. 12-14, 2018.

Jon M. Percaz, Israel Arnedo, Ivan Arregui, Fernando Teberio, Miguel A. G. Laso, and Txema Lopetegi are with the Electrical, Electronic and
Communications Engineering Department, Public University of Navarre, 31006 Pamplona, Spain. e-mail: jon.percaz@unavarra.es.

P. Martin-Iglesias is with the European Space Agency ESA-ESTEC, 2201 AZ Noordwijk, The Netherlands. 
excitation of parasitic modes (including reflection) in linear tapers is often relatively high, and to reduce it the length of the taper must be increased often to large values. A better taper design was achieved by successive connection of several cones with different contour angles [20], [21]. However, when the number of conical sections employed is large, these tapers approach the superior nonlinear smooth tapers, where the contour angle is changed continuously. Several techniques have been proposed to design nonlinear smooth tapers, most of them resting on the use of the coupled-mode theory [15], [16]. Some elegant and solid synthesis procedures are available for circular waveguide tapers [22]-[24], [15], [16], also applicable to rectangular tapers where the height of the waveguide is kept constant [15]. Guidelines to extend the synthesis procedures to arbitrary cross sections are given in [16] and an analytical solution for parabolic tapers is obtained in [25]. All these synthesis procedures achieve nonlinear smooth tapers for overmoded (highly multimode) waveguides, with very low excitation of the parasitic mode, where only the most strongly coupled unwanted (parasitic) mode is taken into account, and assuming that both modes are far above cutoff. These devices are of high interest in the field of high-power microwaves or to transmit microwave signals with very low losses. If several higher-order parasitic modes need to be taken into account in the synthesis, then a quasidiagonalization of the coupled-mode equations can be performed [26], [27], [15]. Using this technique a more accurate synthesis can be achieved but still assuming that all the relevant modes are far above cutoff. More complex taper synthesis techniques (based on the use of horn modes) have been proposed for the case when the parasitic mode is close to cutoff [15], or even when the parasitic mode is below cutoff in a region of the taper [15], [16]. In any case, all these synthesis techniques have been developed assuming multimode operation in the taper and with the aim of minimizing the coupling to the main parasitic mode.

To the authors' knowledge a systematic and general synthesis procedure to design microwave waveguide tapers for singlemode operation able to implement the Klopfenstein and Hecken analytical responses, among others, is not available in the literature. Those single-mode tapers are of high interest for microwave telecommunication devices like filters and couplers. Recently, a novel and interesting design method based on the use of generalized superellipses and optimization algorithms has been proposed, demonstrating successful results in microstrip and rectangular waveguide single-mode tapers [28]. However, a pure synthesis technique able to implement the optimum or near-optimum analytical responses developed for transmission line tapers would be of great interest. This will be broadly the aim of this work. In this paper, a systematic procedure based on the coupled-mode theory to synthetize microwave waveguide tapers for single-mode operation is explained in detail. The technique allows us to implement classical transmission line tapering functions, such as the aforementioned Klopfenstein and Hecken. Additionally, other novel tapering functions, based partially on the frequency response of multisection transformers, will be proposed and implemented. These novel functions will give rise to tapers with smooth profile, retaining the limited bandwidth but also the physical length of the multisection transformers. When properly designed, the limited bandwidth will be adequate for the intended single-mode operation, and the length of the taper can be shorter than that of the Hecken and even the Klopfenstein implementations.

\section{COUPLED-MODE THEORY FOR WAVEGUIDE TAPERS}

As it was explained in detail in [29], [30], the coupled-mode theory, based on the cross-section method [16], can be employed to determine the total electric, $\widehat{\vec{E}}$, and magnetic, $\widehat{\vec{H}}$, fields along a general nonuniform waveguide structure, with a cross-section that varies smoothly in the propagation direction.

Additionally, if single-mode operation is assumed, the complex amplitudes of the forward and backward travelling waves associated to the mode, $a^{+}$and $a^{-}$respectively, are determined just by a simple coupled-mode equation system, (1.a) and (1.b), that is governed by the coupling coefficient, $K$, between those waves [29], [30]:

$$
\begin{aligned}
& \frac{d a^{+}}{d z}=-j \cdot \beta \cdot a^{+}+K \cdot a^{-} \\
& \frac{d a^{-}}{d z}=j \cdot \beta \cdot a^{-}+K \cdot a^{+}
\end{aligned}
$$

where:

$$
\begin{aligned}
& \widehat{\vec{E}}=a^{+} \cdot \vec{E}^{+}+a^{-} \cdot \vec{E}^{-} \\
& \widehat{\vec{H}}=a^{+} \cdot \vec{H}^{+}+a^{-} \cdot \vec{H}^{-}
\end{aligned}
$$

being $\vec{E}^{+}, \vec{H}^{+}$and $\vec{E}^{-}, \vec{H}^{-}$the vector mode patterns of the forward (+) and backward (-) traveling waves; $z$, the propagation direction; and $\beta$, the propagation constant.

A general expression for the coupling coefficient, $K$, valid for nonuniform waveguide structures where the dimensions of the metallic cross-section vary along the propagation direction, can be given as [16], [29]:

$$
K=\frac{-\pi \cdot f \cdot \oint v \cdot\left\{\mu_{0} \cdot\left[\left(H_{z}^{+}\right)^{2}+\left(H_{t}^{+}\right)^{2}\right]+\varepsilon \cdot\left(E_{n}^{+}\right)^{2}\right\} \cdot d t}{2 \cdot N^{+} \cdot \beta}
$$

where the line integral is performed over the metallic contour of the cross-section (the contour of intersection between the metaldielectric interface and the cross section of the waveguide); $f$ is the frequency; $\mu_{0}$, the magnetic permeability; $\varepsilon$, the electrical permittivity; and $v=\tan (\alpha)$, the tangent of the angle $\alpha$ defined between the $z$ axis and the line tangential to the metal-dielectric interface and orthogonal to the metallic contour of the crosssection. The definition of the unit vectors $\hat{n}, \hat{t}, \hat{z}$ that make up the system of axes is as follows: $\hat{t}$ is tangential to the metallic contour of the cross-section; $\hat{n}$ is normal to $\hat{t}$, placed in the crosssection plane and directed towards the metal; and $\hat{z}$, is in the direction of the waveguide axis. For more details, see [16], [29]. Moreover, $\mathrm{H}_{z}^{+}$and $\mathrm{H}_{t}^{+}$are the $z$ and $t$ components, respectively, of the magnetic field of the forward travelling wave associated to the propagation mode, whereas $E_{n}^{+}$is the $n$ component of the electric field of the same wave. Finally, $N^{+}$, is defined as the mode normalization factor that can be calculated as: 


$$
N^{+}=\iint_{S}\left(\vec{E}^{+} \times \vec{H}^{+}\right) \cdot d \vec{S}
$$

where $S$ is the surface of the waveguide cross-section.

In the case of a tapered matching section, it is worth noting that there is an inherent port mismatch level, $\rho_{0}=S_{11}(\beta=0)$, whose specific value will depend on the difference between the cross-sections of its ports. Following the notation of (2), $\rho_{0}$ can be defined as:

$$
\rho_{0}=S_{11}(\beta=0)=\left.\frac{a^{-}(z=0)}{a^{+}(z=0)}\right|_{a^{-}(z=L)=0}
$$

where $L$ is the length of the taper. Taking into account the $\rho_{0}$ definition of (5), and setting $\beta=0$ and $z=0$ in the coupledmode equations (1), a useful relationship between $\rho_{0}$ and $K$ can be obtained after some mathematical manipulations:

$$
\rho_{0}=\tanh \left[-\int_{0}^{L} K(z) \cdot d z\right]
$$

To finish this section, it is interesting to note that the coupling coefficient, $K(z)$, depends finally on the physical dimensions of the waveguide and, therefore, for a target coupling coefficient obtained through a synthesis method, the waveguide physical parameters can be extracted [16], [29], [30].

\section{SyNTHESIS OF CLASSICAL AND NEW TAPER SOLUTIONS USING THE COUPLED-MODE THEORY}

The fundamentals of the coupled-mode theory for tapered matching sections have been presented in the previous section, and now a synthesis procedure for waveguide tapers will be explained in detail.

\section{A. Exact Inverse Scattering Synthesis Technique for Tapers}

The synthesis method is based on the use of an exact series solution of the inverse scattering problem that was originally presented in [31], [32] and successfully employed in many other cases [33]-[35].

The basic principle of this technique is that in order to synthetize a microwave device characterized by a target frequency response in reflection, $S_{11}(\beta)$, only limited by the principles of causality, passivity and stability, the required coupling coefficient, $K(z)$, can be calculated by means of (7):

$$
\begin{aligned}
K(z)= & -2 \cdot F(2 z)-2 \int_{0}^{2 z} d x_{1} F\left(x_{1}\right) \int_{0}^{x_{1}} d x_{2}^{\prime} F\left(x_{2}^{\prime}\right) \\
& \times F\left(x_{2}^{\prime}-x_{1}+2 z\right)-2 \int_{0}^{2 z} d x_{1} F\left(x_{1}\right) \\
& \times \int_{0}^{x_{1}} d x_{2}^{\prime} F\left(x_{2}^{\prime}\right) \int_{0}^{x_{2}} d x_{3}^{\prime} F\left(x_{3}^{\prime}\right) \int_{0}^{x_{3}} d x_{4}^{\prime} F\left(x_{4}^{\prime}\right) \\
& \times F\left(x_{4}^{\prime}-x_{3}+2 z\right)-\cdots-2 \int_{0}^{2 z} d x_{1} F\left(x_{1}\right) \\
& \times \int_{0}^{x_{1}} d x_{2}^{\prime} F\left(x_{2}^{\prime}\right) \int_{0}^{x_{2}} \cdots \int_{0}^{x_{2 N-1}} d x_{2 N}^{\prime} F\left(x_{2 N}^{\prime}\right) \\
& \times F\left(x_{2 N}^{\prime}-x_{2 N-1}+2 z\right)-\cdots-
\end{aligned}
$$

being $x_{i}^{\prime}=x_{i}+x_{i-1}-2 z$ for $i>1$ and:

$$
F(\tau)=\frac{1}{2 \pi} \cdot \int_{-\infty}^{\infty} S_{11}(\beta) \cdot e^{j \beta \tau} \cdot d \beta
$$

It is important to highlight that the exact series solution of (7) is valid for single mode operation, while in (8), the propagation constant, $\beta$, is assumed to be independent of the position along the propagation direction, $z$.

When (7) is numerically calculated, the infinite series has to be truncated and we say that the order of approximation of the solution is $N$ when the number of terms used is $N+1$. The higher the order $N$, the better the accuracy, being the high reflectivity cases those that require the highest orders [32]. Fortunately, the situation here is of low reflectivity, because a taper usually requires $\left|S_{11}\right| \leq \rho_{m}$ in the bandwidth of interest, with $\rho_{m}$ defined as:

$$
\rho_{m}=10^{-R L / 20}
$$

where $R L$ (in $\mathrm{dB}$ ) is the return loss level required for the taper. Since the intended $R L$ level for a taper is typically high, $\rho_{m}$ will be small, and a low reflectivity response is usually needed. This allows us to calculate the required coupling coefficient with good accuracy using a low order of approximation for the series, making this synthesis method very suitable for tapers.

\section{B. Synthesis of the Classical Klopfenstein and Hecken Tapers Based on the Coupled-Mode Theory}

Taking advantage of the coupled-mode theory presented in Section II, together with the exact inverse scattering synthesis technique of Section III.A, classical Klopfenstein and Hecken tapers can be synthetized for transmission line and waveguide technologies. In essence, both tapers were originally developed for an ideal transmission line model [6], [10] and, consequently, they were intended to match two transmission lines of different characteristic impedances, $Z_{p 1}$ and $Z_{p 2}$, being $Z_{p 1}$ the characteristic impedance of the incoming transmission line to the taper (port 1) and $Z_{p 2}$ the characteristic impedance of the outcoming transmission line (port 2).

Focusing on the Klopfenstein classical taper solution, its ideal frequency response in reflection exhibits an equiripple behavior with a maximum reflection level of $\rho_{m}$, when the propagation constant is higher than a given value, $\beta_{\text {min }}$, as depicted in Fig. 1. Its frequency response can be expressed analytically as a function of the propagation constant, $\beta$, as [36], [6]:

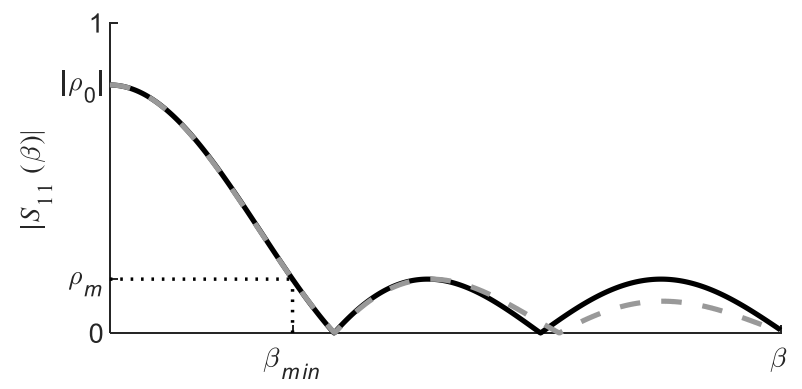

Fig. 1. $\left|S_{11}\right|$-parameter as a function of the propagation constant for classical tapers: Klopfenstein (black solid line) and Hecken (grey dashed line). 


$$
S_{11}(\beta)=\rho_{0} \cdot \frac{\cos \left(\sqrt{(\beta L)^{2}-A^{2}}\right)}{\cosh (A)} \cdot e^{-j \beta L}
$$

where $A$ is given in (11) and $L$ is the taper length.

$$
A=\cosh ^{-1}\left(\frac{\left|\rho_{0}\right|}{\rho_{m}}\right)
$$

An analogous expression for $S_{11}(\beta)$ is also presented in [36], deduced from [10], for the case of the Hecken taper, see (12). As it is shown in Fig. 1, the frequency response of the Hecken taper is somewhat different from that of the Klopfenstein taper. In particular, the $\left|S_{11}(\beta)\right|$ of the Hecken taper exhibits a decreasing (not equiripple) maximum reflection level beyond $\beta_{\min }$, where the maximum in-band reflection $\rho_{m}$ is reached.

$$
S_{11}(\beta)=\rho_{0} \cdot \frac{B}{\sinh (B)} \cdot \frac{\sin \left(\sqrt{(\beta L)^{2}-B^{2}}\right)}{\sqrt{(\beta L)^{2}-B^{2}}} \cdot e^{-j \beta L}
$$

The $B$ parameter in (12) can be calculated by using (13) to achieve the required $\rho_{m}$ :

$$
\frac{B}{\sinh (B)} \cdot 0.21723=\frac{\rho_{m}}{\left|\rho_{0}\right|}
$$

If a transmission line model is used, the coupling coefficient, $K(z)$, is related to the characteristic impedance of the taper along the propagation direction, $Z_{0}(z)$, through the expression [31]:

$$
K(z)=-\frac{1}{2 \cdot Z_{0}(z)} \cdot \frac{d Z_{0}(z)}{d z}
$$

Substituting this last expression into (6), we obtain:

$$
\rho_{0}=\tanh \left[-\int_{0}^{L} K(z) \cdot d z\right]=\tanh \left[\frac{1}{2} \cdot \ln \left(\frac{Z_{p 2}}{Z_{p 1}}\right)\right]
$$

Taking into account that $\tanh (x)=\left(e^{x}-e^{-x}\right) /\left(e^{x}+e^{-x}\right)$, and after some mathematical manipulations, the familiar expression for $\rho_{0}$ as a function of the characteristic impedances of the ports, can be deduced from (15):

$$
\rho_{0}=\frac{Z_{p 2}-Z_{p 1}}{Z_{p 2}+Z_{p 1}}
$$

Now, in order to calculate the required coupling coefficient for the Klopfenstein and Hecken taper responses, a $N=0$ (0-th order) approximation of the exact series solution of the synthesis problem (7) is taken. As it was demonstrated in [36], this is equivalent to the low reflectivity approximation that was employed in [6], [10]. By following this procedure, an analytical expression for $K(z)$ will be obtained for both cases. Thus, the coupling coefficient for the Klopfenstein taper results in (17) as it was presented in [36], deduced from [6]:

$$
\begin{aligned}
K(z)=\frac{-\rho_{0}}{\cosh (A)} \cdot\left[\frac{A^{2}}{L} \cdot \frac{I_{1}\left(A \cdot \sqrt{1-\left(\frac{z-L / 2}{L / 2}\right)^{2}}\right)}{A \cdot \sqrt{1-\left(\frac{z-L / 2}{L / 2}\right)^{2}}}\right. \\
+\frac{1}{2} \cdot \delta(z)+\frac{1}{2} \cdot \delta(z-L)
\end{aligned}
$$

where $I_{1}(x)$ is the modified Bessel function of the first kind of first order. The impulse functions $\delta(z)$ and $\delta(z-L)$ present in the coupling coefficient will produce the characteristic step discontinuities at the beginning and at the end of the Klopfenstein taper profile.

In the same way, the expression of $K(z)$ for the Hecken taper results in (18), as it was presented in [36], deduced from [10]:

$$
K(z)=-\rho_{0} \cdot \frac{B / L}{\sinh (B)} \cdot I_{0}\left[B \cdot \sqrt{1-\left(\frac{z-L / 2}{L / 2}\right)^{2}}\right]
$$

where $I_{0}(x)$ is the modified Bessel function of the first kind of zero order.

It must be noted that the use of the 0-th order (low reflectivity) approximation leads to an inaccuracy in the synthesized coupling coefficient because, if we take the actual $\rho_{0}$ value, the taper will not reach $Z_{p 2}$ at $z=L$, with $Z_{0}(z)$ being calculated by (14), as it was shown in [36]. In order to overcome this issue, the actual value of $\rho_{0}$ has to be overestimated and given by its 0 -th order (low reflectivity) approximation, $\rho_{0}^{\prime}$, which can be obtained by performing the Fourier transform of (7) for $N=0$, leading to [37]:

$$
\rho_{0}^{\prime}=\left.S_{11}(\beta=0)\right|_{N=0}=-\int_{0}^{L} K(z) \cdot d z
$$

Now, if we substitute (14) into (19), we will obtain $\rho_{0}^{\prime}$ as a function of $Z_{p 1}$ and $Z_{p 2}$, with an expression identical to that proposed in [6], [10] for an ideal transmission line model:

$$
\rho_{0}^{\prime}=\frac{1}{2} \cdot \ln \left(\frac{Z_{p 2}}{Z_{p 1}}\right)
$$

Hence, $\rho_{0}$ in the synthesis equations (11), (13), (17) and (18), must be substituted by the new overestimated $\rho_{0}^{\prime}$ in order to guarantee that the taper achieves the intended impedance values or cross-section dimensions at its ports. Once the suitable synthesis parameters are known, the resulting length of the Klopfenstein taper will be determined by $L=A / \beta_{\text {min }}$, whereas for the Hecken taper case will be $L=\sqrt{B^{2}+6.523} / \beta_{\text {min }}$.

To finish this subsection it is worth noting that the inaccuracy of the low reflectivity approximation in these classical tapers 
was surpassed in [36] by employing a higher $\operatorname{order} N>0$ in the series solution of the synthesis. Unfortunately, this solution results in an increase of the taper length that is not practical and has been discarded in this work.

\section{New Taper Solutions Based on Multisection Quarter- Wave Chebyshev Transformers}

The use of multisection quarter-wave matching transformers is a classical method to achieve impedance matching between two different transmission lines [4], [5], [38].

The multisection Chebyshev transformer consists of $M$ commensurate transmission lines in cascade, all of them with the same electrical length, but with different characteristic impedances $Z_{i}$ (with $1 \leq i \leq M$ ), featuring a Chebyshev frequency response of order $M$. The commensurate lines have the same frequency behavior due to their identical electrical lengths. In fact, all lines have ideally the same physical length, $l$, and show the same wavelength, $\lambda_{g}$, and propagation constant, $\beta$, at any particular frequency. These properties allow us to define the frequency behavior of the prototype as a function of the electrical length of the line, $\theta$, or equivalently as a function of $\beta$ :

$$
\theta=\beta \cdot l=\frac{2 \cdot \pi}{\lambda_{g}} \cdot l
$$

In order to extend the validity of the multisection Chebyshev transformer prototype to general waveguide technologies, we can employ equivalent characteristic impedances for the ports, $Z_{p 1}, Z_{p 2}$, defined in such a way that the $\rho_{0}$ achieved by them (calculated with (16)) is identical to the actual $\rho_{0}$ produced by the waveguide ports mismatch, as calculated by (6). If we set $Z_{p 1}=1 \Omega$ for convenience, we can obtain $Z_{p 2}$ from (16) as:

$$
Z_{p 2}=\frac{1+\rho_{0}}{1-\rho_{0}}
$$

Once the equivalent impedances, $Z_{p 1}$ and $Z_{p 2}$, are defined (or the actual values are known for the case of transmission line technologies), the normalized characteristic impedance of each commensurate line, $Z_{i}$, can be determined by applying the wellknown Richards' transformation and the iterative extraction procedure fully detailed in [39]. Alternative calculation methods for the $Z_{i}{ }^{\prime} s$ are explained in [4], [5], [38], although they are less convenient when the order $M$ of the multisection transformer is increased. The values of the $Z_{i}{ }^{\prime} s$ can be also calculated using

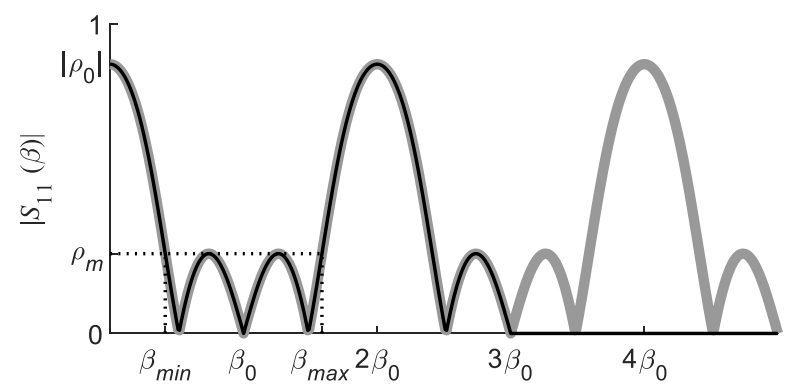

Fig. 2. $\left|S_{11}\right|$-parameter as a function of the propagation constant for a classical $M=3$ (3-rd order) multisection quarter-wave Chebyshev transformer (grey line) and an example of target response for the novel taper to be synthetized with $\beta_{z}=3 \cdot \beta_{0}$ (black line). commercial software synthesis tools like S/Filsyn.

Now we can obtain the $S_{11}(\beta)$ of the transformer prototype by means of the [ABCD] matrix, just by cascading the $Z_{i}$ transmission line sections (multiplying their matrices) and loading the output port with $Z_{p 2}$, employing $Z_{p 1}$ as the reference impedance. As it is shown in Fig. 2, the frequency response $S_{11}(\beta)$ has a periodic behavior which repeats every $2 \cdot \beta_{0}$. The matched bandwidth is centered at $\beta_{0}$, that is the propagation constant value at which the electrical length of the commensurate lines is $\theta_{0}=\beta_{0} \cdot l=\pi / 2$, leading to $l=\lambda_{g 0} / 4$, with $\lambda_{g 0}=(2 \cdot \pi) / \beta_{0}$.

The lower and upper limits of the propagation constant range, $\beta_{\text {min }}$ and $\beta_{\text {max }}$, that complies with the required $\rho_{m}$ level for a $M$-th order multisection Chebyshev transformer can be calculated using (23) and (24):

$$
\begin{gathered}
\beta_{\text {min }}=\beta_{0} \cdot \frac{2 \cdot \theta_{m}}{\pi} \\
\beta_{\text {max }}=2 \cdot \beta_{0} \cdot\left(1-\frac{\theta_{m}}{\pi}\right)
\end{gathered}
$$

where $\theta_{m}=\cos ^{-1}\left\{\operatorname{sech}\left[\frac{1}{M} \cdot \cosh ^{-1}\left(\frac{\left|\rho_{0}\right|}{\rho_{m}} \cdot \sqrt{\frac{1-\rho_{m}^{2}}{1-\rho_{0}^{2}}}\right)\right]\right\}$

When obtaining the multisection transformer prototype, it is convenient to consider a transformer whose $\beta_{\text {min }}$ is as close as possible to the required $\beta_{\text {low }}$ for the taper, while fulfilling also the required matched bandwidth. This election will eventually lead to the shortest taper. When a valid multisection transformer prototype is found, the procedure proposed in [40] to achieve a fully smooth device from a periodic $S_{11}(\beta)$, as the one obtained here, can be applied. The method requires just to set the $S_{11}(\beta)$ to zero beyond a certain $\beta_{z}$, retaining just several full periods of the frequency response (with the basic period defined symmetric around $\beta=0$ ). This method will allow us to synthetize fully smooth tapered matching sections, by applying the exact series solution of Section III.A to the obtained $S_{11}(\beta)$, while maintaining a physical length similar to that of the initial multisection Chebyshev transformer prototype.

\section{SyNTHESIS STRATEGY FOR NONUNIFORM WAVEGUIDES WHERE $\beta$ VARIES ALONG THE PROPAGATION DiRECTION}

As it has been stated in Section III.A, the synthesis technique employed is only valid for waveguides where the propagation constant does not vary along the propagation direction and only depends on frequency, i.e., $\beta=\beta(f)$.

However, when a taper is required, in many cases a change of the electrical or geometrical properties of the waveguide is involved, leading to an implicit change of the propagation constant along the propagation direction, i.e., $\beta=\beta(f, z)$. In this case, it is necessary to introduce an average propagation constant, $\bar{\beta}(f)$, that does not vary along a normalized propagation direction, $\chi$. For the mathematical formulation to be consistent, the differential electrical length achieved along a certain $d \chi$, i.e., $\bar{\beta} \cdot d \chi$, must be the same caused by the actual $\beta$ in $d z$, i.e., $\bar{\beta} \cdot d \chi=\beta \cdot d z$. This relationship implies that:

$$
\frac{d z}{d \chi}=\frac{\bar{\beta}}{\beta}
$$


In view of (25), we can calculate the actual propagation axis $z$ from the normalized axis $\chi$, by means of (26):

$$
z(\chi)=\int_{0}^{\chi} \frac{\bar{\beta}}{\beta} \cdot d r
$$

where $r$ is a dummy variable.

Let's rewrite now the first coupled-mode equation (1.a) as a function of the normalized propagation axis $\chi$, and of the average propagation constant, $\bar{\beta}$. To do it, we rewrite:

$$
\frac{d a^{+}}{d z}=\frac{d a^{+}}{d \chi} \cdot \frac{d \chi}{d z}
$$

Proceeding in the same way, and taking into account the dependence of the coupling coefficient, $K(z)$, on $v$, see (3), with $v=\tan (\alpha)$ calculated as the derivative with $\mathrm{z}$ of the corresponding physical dimension, it will be possible to rewrite:

$$
K(z)=K(\chi) \cdot \frac{d \chi}{d z}
$$

Moreover, taking into account (25), $\beta$ can be also expressed as a function of $\bar{\beta}$ and $d \chi / d z$ :

$$
\beta=\bar{\beta} \cdot \frac{d \chi}{d z}
$$

Now, by substituting (27), (28) and (29) into (1.a), we can finally obtain (30.a). If we proceed in a similar manner with (1.b), we can find (30.b):

$$
\begin{aligned}
& \frac{d a^{+}}{d \chi}=-j \cdot \bar{\beta} \cdot a^{+}+K(\chi) \cdot a^{-} \\
& \frac{d a^{-}}{d \chi}=j \cdot \bar{\beta} \cdot a^{-}+K(\chi) \cdot a^{+}
\end{aligned}
$$

It is important to highlight that (30.a) and (30.b) constitute a system of coupled-mode equations, fully analogous to (1.a)(1.b), but formulated for the normalized position $\chi$, and the average propagation constant $\bar{\beta}$, that does not vary with $\chi$. This allows us to perform the taper synthesis by means of the method detailed in Section III.A, but employing $\bar{\beta}$ and $\chi$ instead of $\beta$ and $z$, and calculating afterwards $z(\chi)$ by (26), in the last step of the synthesis process, after the physical dimensions of the waveguide taper have been determined. By doing so, the synthetized device finally achieved is fully suitable for a position dependent $\beta$. Nevertheless, it must be noted that the $z(\chi)$ transformation is done for a single frequency, $f_{t}$, and it works properly when the quotient $\bar{\beta}(f) / \beta(f, z)$ does not vary much with respect to $\bar{\beta}\left(f_{t}\right) / \beta\left(f_{t}, z\right)$. Due to this fact, it is advisable to perform the transformation for a $f_{t}$ located at the center of the operation bandwidth of the taper.

Since the waveguide dimensions at the beginning $(z=0)$ and at the end $(z=L)$ of the taper will be typically a given requirement, we can define $\bar{\beta}(f)$ as:

$$
\bar{\beta}(f)=\frac{\beta(f, z=0)+\beta(f, z=L)}{2}
$$

Not only for the propagation axis transformation, but the average propagation constant will be also relevant when the specifications of the starting prototype are defined (both for the classical tapers and for the novel tapers proposed in this paper). Actually, as it will be shown, we need to consider $K\left(z, \bar{\beta}_{\text {min }}\right)$, with $\bar{\beta}_{\text {min }}=\bar{\beta}\left(f=f_{\text {min }}\right)$, where $f_{\text {min }}$ is the minimum frequency that is required to satisfy the $\rho_{m}$ level, in order to extrapolate the $\rho_{0}$ and $\rho_{0}^{\prime}$ levels (through (6) and (19), respectively) that would correspond to $S_{11}(\beta=0)$ if $K(z)$ were constant with $\beta(f)$. Therefore, when the taper is designed for a waveguide where $\beta$ varies along the propagation direction, we need to define the requirements of $\rho_{0}$ and $\rho_{0}^{\prime}$ for $\bar{\beta}_{\text {min }}$ (or its corresponding $f_{\text {min }}$ ) in order to synthetize a suitable frequency response, $S_{11}(\bar{\beta})$, that will eventually meet the expected performance. Moreover, this criterion must be followed also when relating $K(z)$ with the physical dimensions of the waveguide because, if it is not, the waveguide cross-section achieved at $z=L$ will not result in the required one for the output port.

\section{Rectangular WaVeguide: a SPeCific ApPlication TECHNOLOGY}

The series solution shown in Section III.A provides a general method to calculate the coupling coefficient for both classical and novel tapers. However, in order to extract the taper physical dimensions we must choose a specific waveguide technology, and establish the relationship between physical parameters and coupling coefficient. In this paper, we will show examples in rectangular waveguide, since it is one of the most widely employed waveguide technologies.

As it was shown in [29], the coupling coefficient of the rectangular waveguide for single mode operation exhibits an analytical expression that depends on its width and height, $a(z)$ and $b(z)$ respectively, and on the propagation constant, $\beta$ :

$$
K(z)=\frac{-1}{2 \cdot b(z)} \cdot \frac{d b(z)}{d z}+\frac{\pi^{2}}{2 \cdot a(z)^{3} \cdot \beta^{2}} \cdot \frac{d a(z)}{d z}
$$

where $\beta$ is the propagation constant of the $T E_{10}$ fundamental mode that can be calculated by:

$$
\beta=\frac{2 \cdot \pi \cdot f}{c} \cdot \sqrt{1-\left(\frac{c}{2 \cdot a(z) \cdot f}\right)^{2}}
$$

being $c=c_{0} / \sqrt{\varepsilon_{r}}$, with $c_{0}$ the speed of light in vacuum, and $\varepsilon_{r}$ the relative electrical permittivity that fills the inner volume of the rectangular waveguide. It is worth noting that $K(z)$ will exhibit an inherent dependence with frequency (32), since the propagation constant, $\beta$, depends on it (33).

In order to set the requirements of a taper, the first task is to determine $\rho_{0}$ just by introducing (32) in (6) for the case of rectangular waveguide. The final solution for $\rho_{0}$ is shown in (34), revealing that it can be calculated just considering the required values of $a(z)$ and $b(z)$ at $z=0$ and $z=L$ : 


$$
\rho_{0}=\tanh \left\{\frac{1}{4} \ln \left[\left(\frac{b(L)}{b(0)}\right)^{2} \cdot \frac{1-\left(\frac{c}{2 \cdot f_{\min } \cdot a(0)}\right)^{2}}{1-\left(\frac{c}{2 \cdot f_{\min } \cdot a(L)}\right)^{2}}\right]\right\}
$$

Regarding the 0 -th order (low reflectivity) approximation of $\rho_{0}^{\prime}$, which is employed for the case of Klopfenstein and Hecken tapers, it can be calculated by introducing (32) in (19), again for rectangular waveguide, resulting in:

$$
\rho_{0}^{\prime}=\frac{1}{4} \ln \left[\left(\frac{b(L)}{b(0)}\right)^{2} \cdot \frac{1-\left(\frac{c}{2 \cdot f_{\min } \cdot a(0)}\right)^{2}}{1-\left(\frac{c}{2 \cdot f_{\min } \cdot a(L)}\right)^{2}}\right]
$$

Furthermore, if we pay attention to (32), it is clear that $K(z)$ can be divided into two parts with respect to the dimensions of the rectangular waveguide. The left side summand is only a function of $b(z)$, while the right one has an exclusive dependency with $a(z)$. This allows us to rewrite $K(z)$ as $K(z)=K_{b}(z)+K_{a}(z)$ with $K_{b}(z)$ and $K_{a}(z)$ as in (36) and (37):

$$
\begin{gathered}
K_{b}(z)=\frac{-1}{2 \cdot b(z)} \cdot \frac{d b(z)}{d z}=k_{b} \cdot K(z) \\
K_{a}(z)=\frac{\pi^{2}}{2 \cdot a(z)^{3} \cdot \beta_{\min }{ }^{2}} \cdot \frac{d a(z)}{d z}=k_{a} \cdot K(z)
\end{gathered}
$$

where $k_{b}$ and $k_{a}$ are the constants that control which part of the general coupling coefficient $K(z)$ is performed by $K_{b}(z)$ and $K_{a}(z)$. The expressions to calculate both constants, $k_{b}$ and $k_{a}$, are available in (38) and (39), and they exclusively depend on the dimensions of the cross-sections at $z=0$ and $z=L$, as expected.

$$
\begin{gathered}
k_{b}=\frac{\int_{0}^{L} K_{b}(z) \cdot d z}{\int_{0}^{L} K(z) \cdot d z}=\frac{-\frac{1}{2} \cdot \ln \left[\frac{b(z=L)}{b(z=0)}\right]}{-\rho_{0}^{\prime}} \\
k_{a}=\frac{\int_{0}^{L} K_{a}(z) \cdot d z}{\int_{0}^{L} K(z) \cdot d z}=\frac{\frac{1}{4} \cdot \ln \left[\frac{1-\left(\frac{c}{2 \cdot f_{\min } \cdot a(L)}\right)^{2}}{1-\left(\frac{c}{2 \cdot f_{\min } \cdot a(0)}\right)^{2}}\right]}{-\rho_{0}^{\prime}}
\end{gathered}
$$

where $\rho_{0}^{\prime}$ is given in (35). It is interesting to note that $k_{b}$ and $k_{a}$ satisfy $k_{b}+k_{a}=1$, guaranteeing that $K_{b}(z)+K_{a}(z)=k_{b}$. $K(z)+k_{a} \cdot K(z)=K(z)$.

The $b(z)$ profile of the rectangular waveguide taper can be calculated with the expression (40) obtained in [29], whereas the $a(z)$ profile can be calculated by employing (41):

$$
b(z)=b(0) \cdot e^{-2 \cdot \int_{0}^{z} K_{b}(r) \cdot d r}
$$

$$
a(z)=\frac{\frac{c}{2 \cdot f_{\min }} \cdot e^{-2 \cdot \int_{0}^{z} K_{a}(r) \cdot d r}}{\sqrt{e^{-4 \cdot \int_{0}^{z} K_{a}(r) \cdot d r}+\left(\frac{c}{2 \cdot a(0) \cdot f_{\min }}\right)^{2}-1}}
$$

where $r$ in (40) and (41) is a dummy variable.

Finally, it must be highlighted that if we want to synthetize a taper that features a variable width, i.e., $k_{a} \neq 0$, we will need to apply the synthesis strategies explained is Section IV because of the variation of the propagation constant with the position.

The robustness and flexibility of the proposed taper synthesis method will be verified by synthetizing tapers fulfilling certain frequency specifications, between two rectangular waveguides, in three different scenarios: mismatch in cross-section heights, widths and both heights and widths simultaneously.

\section{A. Rectangular Waveguide Tapers Implemented with Variations in Height Only}

The possibility of synthetizing tapers by means of the coupled-mode theory with exclusive variation in the height of the waveguide cross sections is going to be demonstrated for each of the following cases: a Klopfenstein taper, a Hecken taper, and a novel taper based on a multisection Chebyshev transformer. In all cases, they must interconnect a standard WR90 port with a waveguide with the half of that height and the same width while achieving $R L \geq 40 \mathrm{~dB}\left(\rho_{m}=0.01\right.$ by (9)) for the whole WR90 frequency range between $f_{\text {low }}=8.2 \mathrm{GHz}$ and $f_{u p}=12.4 \mathrm{GHz}$. In order to obtain the shortest tapers, we will choose $f_{\text {min }}=f_{\text {low }}$.

Firstly, we need to calculate the mismatch $\rho_{0}$ caused by the height difference. The waveguide height of the WR90 standard is $b(0)=10.16 \mathrm{~mm}$ and, thus, $b(L)=b(0) / 2=5.08 \mathrm{~mm}$. Applying (34), $\rho_{0}=-1 / 3$, while $\rho_{0}^{\prime}=-0.3465$ by (35). Finally, considering that the width of the WR90 standard is $a=22.86$ $\mathrm{mm}$, we can calculate the corresponding propagation constant values for $\beta_{\text {min }}=\beta\left(f_{\text {min }}\right)=103.19 \mathrm{rad} / \mathrm{m}$. and $\beta\left(f_{u p}\right)=$ $220.58 \mathrm{rad} / \mathrm{m}$.

Since $\rho_{0}^{\prime}, \rho_{m}$, and $\beta_{\text {min }}$ are known, the coupling coefficient, $K(z)$, for the Klopfenstein taper can be immediately calculated by means of (17). As we need a taper that matches waveguides with different heights only, then $K_{a}(z)=0$ and, hence, $K_{b}(z)=$ $K(z)$ and, consequently, $k_{b}=1$. Then, if we calculate $b(z)$ using (40), we will obtain the physical dimensions of the Klopfenstein taper with a total length $L=41.07 \mathrm{~mm}$. The coupling coefficient as well as the height profile are depicted in Fig. 3.

Regarding the Hecken taper, we can calculate its $K(z)$ by means of (18). Then, we can follow a reasoning similar to that of the Klopfenstein taper, ensuring $K_{b}(z)=K(z)$ and applying (40) to obtain $b(z)$. Both, $K(z)$ and $b(z)$ for the Hecken taper are shown in Fig. 4. The final length of this taper is $L=47.08$ $\mathrm{mm}$ in this case, larger than the Klopfenstein taper as expected.

The last taper that we are going to consider for waveguides with only height changes is based on the frequency response of a Chebyshev transformer. The first step is to calculate the impedance of the output port assuming an equivalent input port of $Z_{p 1}=1 \Omega$. Considering that $\rho_{0}=-1 / 3$, the impedance for the equivalent output port must be $Z_{p 2}=0.5 \Omega$.

Then, the minimum order, $M$, of the multisection Chebyshev transformer that is capable of meeting the proposed frequency 
specifications must be determined, resulting in $M=4$. The propagation constant $\beta_{0}=178.798 \mathrm{rad} / \mathrm{m}$ at which the commensurate lines exhibits an electrical length of $\theta=\pi / 2 \mathrm{rad}$ is calculated. By using it, the values of $\beta_{\text {min }}=103.19 \mathrm{rad} / \mathrm{m}$ and $\beta_{u p}<\beta_{\text {max }}=254.4 \mathrm{rad} / \mathrm{m}$ are obtained by (23) and (24), respectively.

The calculated equivalent characteristic impedances of each commensurate line are: $Z_{1}=0.934 \Omega, Z_{2}=0.789 \Omega, Z_{3}=0.633$ $\Omega$, and $Z_{4}=0.535 \Omega$. The $S_{11}(\beta)$ of the transformer is calculated

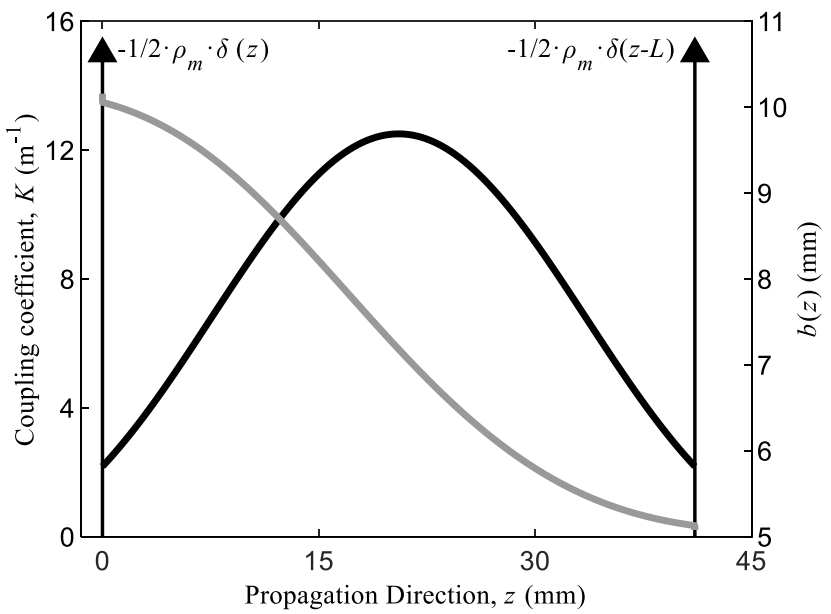

Fig. 3. Coupling coefficient (black line) of the Klopfenstein taper and its rectangular waveguide height variation profile (grey line).

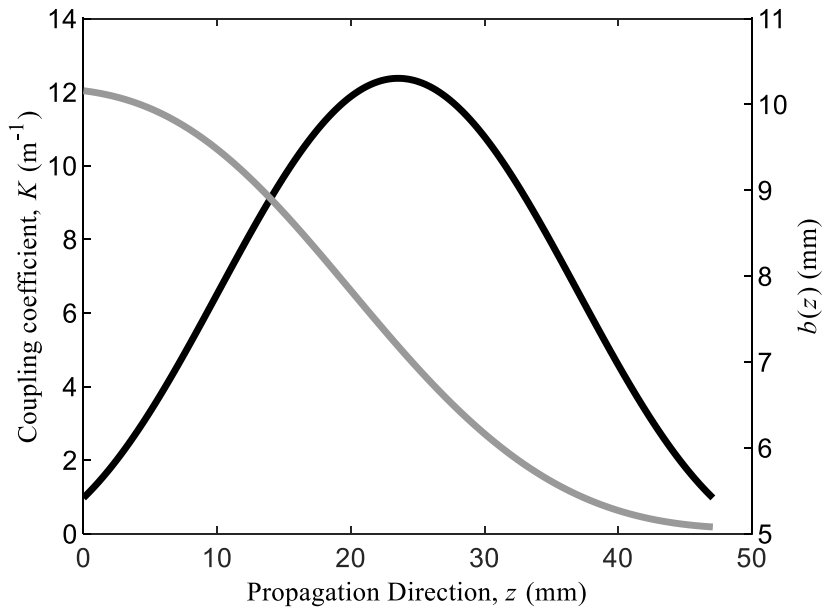

Fig. 4. Coupling coefficient (black line) of the Hecken taper and its rectangular waveguide height variation profile (grey line).

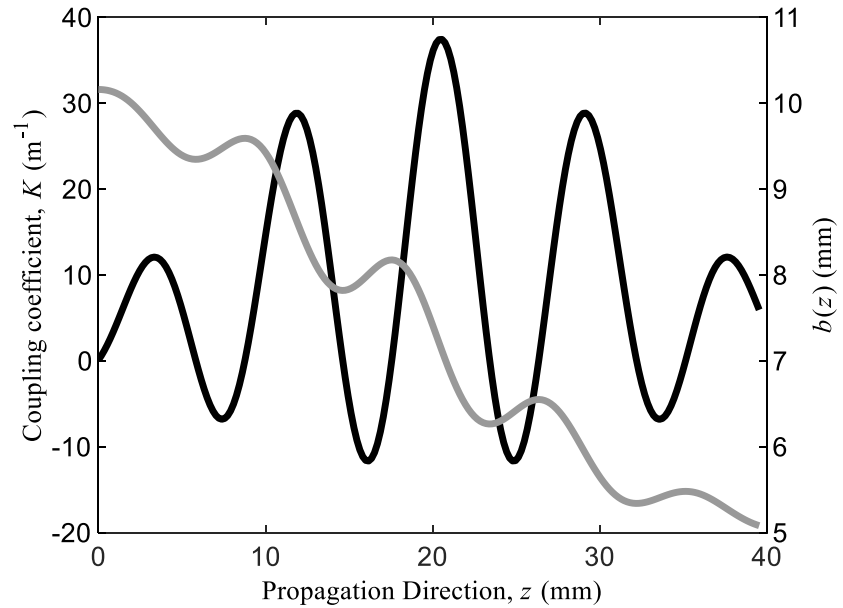

Fig. 5. Coupling coefficient (black line) of the novel taper based on a 4-th order multisection Chebyshev transformer and its rectangular waveguide height variation profile (grey line). by cascading its transmission lines loaded at the end with $Z_{p 2}$, for an input impedance of $Z_{p 1}$, by employing the [ABCD] matrix for every $\beta$ value from $0 \mathrm{rad} / \mathrm{m}$ to $50 \cdot \beta_{0}$ in regular intervals of $\beta_{0} / 50$. Then, the obtained $S_{11}(\beta)$ of the transformer is modified by applying $S_{11}\left(\beta>\beta_{z}\right)=0$ for $\beta_{z}=3 \cdot \beta_{0}$. The obtained $S_{11}(\beta)$ is synthetized using the exact series solution of (7) and (8) with an order $N=4$, see Fig. 5. As for Klopfenstein and Hecken tapers, the physical dimensions of the novel taper are obtained by means of (40) with $K_{b}(z)=k_{b} \cdot K(z)=K(z)$. The height profile, $b(z)$, is also depicted in Fig. 5 .

It must be highlighted that the resulting taper based on a 4-th order multisection Chebyshev transformer has a length $L=$ $39.53 \mathrm{~mm}$, being a bit shorter than Klopfenstein taper that was classically considered as the shortest possible taper solution.

Finally, the synthetized structures were simulated with CST Microwave Studio and the results are depicted in Fig.6 to Fig.8. In all cases, the frequency requirements are achieved, obtaining $R L \geq 40 \mathrm{~dB}$ for the whole frequency range of the WR90 waveguide standard.

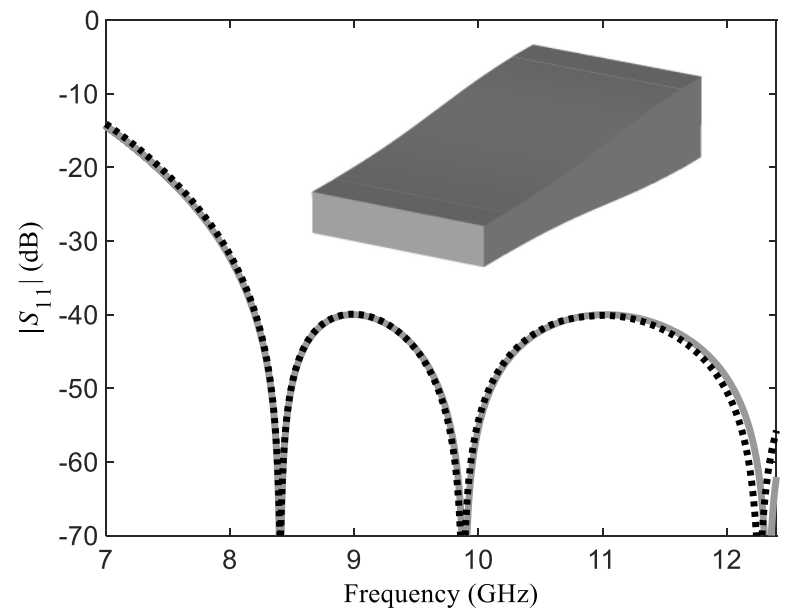

Fig. 6. Comparison of the $\left|S_{11}(f)\right|$-parameters for the Klopfenstein taper: target response (grey solid line) and CST Microwave Studio simulation (black dotted line). Inset: view of the inner hollow volume of the taper.

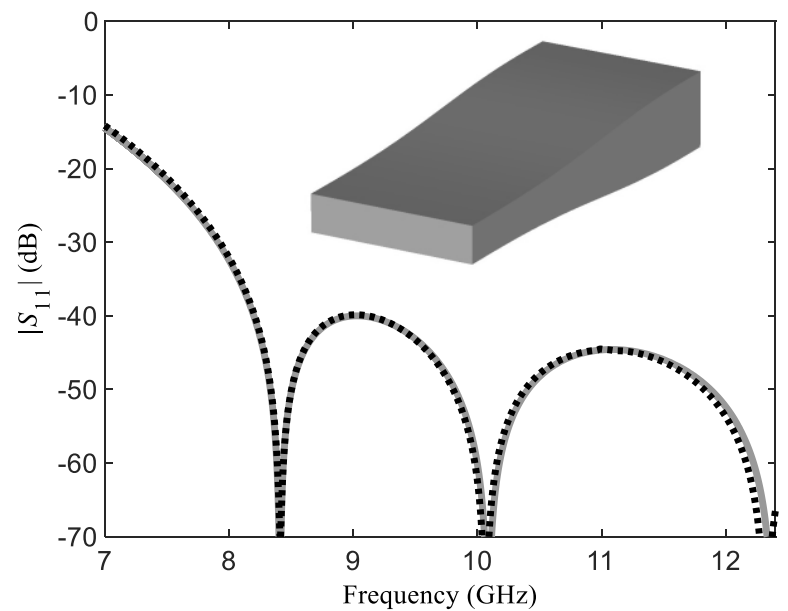

Fig. 7. Comparison of the $\left|S_{11}(f)\right|$-parameters for the Hecken taper: target response (grey solid line) and CST Microwave Studio simulation (black dotted line). Inset: view of the inner hollow volume of the taper. 


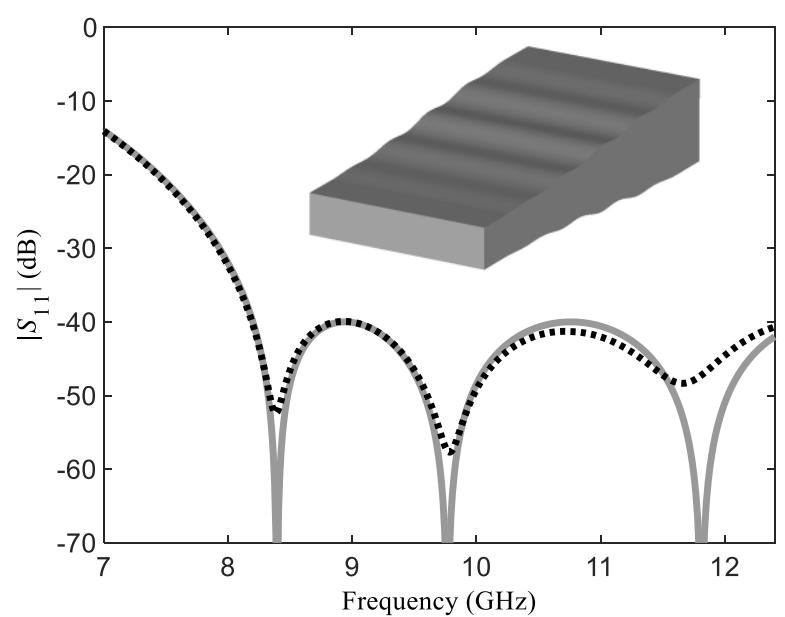

Fig. 8. Comparison of the $\left|S_{11}(f)\right|$-parameters of the novel taper based on a 4-th order multisection Chebyshev transformer: target response (grey solid line) and CST Microwave Studio simulation (black dotted line). Inset: view of the inner hollow volume of the taper.

\section{B. Rectangular Waveguide Tapers Implemented with Variations in Width Only}

The synthesis of rectangular waveguide tapers involving width changes along the propagation direction implies that the propagation constant will depend on the position, as it was detailed in Section IV, where a strategy for dealing with that situation was explained. Taking that into account, two different tapers will be synthetized: a classical Hecken taper and a new taper based on multisection Chebyshev transformers presented for the first time in this paper.

Regarding the dimensions of the taper waveguide ports, the incoming waveguide cross-section width will be the WR90 width, i.e., $a(0)=22.86 \mathrm{~mm}$, and the width of the cross-section of the outcoming waveguide will be the WR62 width, i.e., $a(L)$ $=15.799 \mathrm{~mm}$. The height of the cross section will be fixed to the WR62 height, i.e., $b=7.899 \mathrm{~mm}$. In this case, the required performance of the taper should fulfill $R L$ levels higher than 40 $\mathrm{dB}\left(\rho_{m}=0.01\right.$ by $\left.(9)\right)$ in a frequency range defined from $f_{\text {low }}=f_{\text {min }}=11 \mathrm{GHz}$ up to $f_{\text {up }}=13 \mathrm{GHz}$.

Due to the change of the propagation constant caused by the width variation, we need to consider the propagation constants $\beta(f, z=0)$ and $\beta(f, z=L)$ in order to define the average propagation constant, $\bar{\beta}(f)$. Thus, $\beta(f, z=0)$ is calculated using (33) for $a(0)$, while the value of $\beta(f, z=L)$ is obtained employing $a(L)$. Therefore, for $f=f_{\min }=11 \mathrm{GHz}$, $\beta\left(f_{\text {min }}, z=0\right)=185.1 \mathrm{rad} / \mathrm{m}$ and $\beta\left(f_{\text {min }}, z=L\right)=116.66$ $\mathrm{rad} / \mathrm{m}$ and, consequently, $\bar{\beta}_{\text {min }}\left(f=f_{\text {min }}\right)=150.88 \mathrm{rad} / \mathrm{m}$ by (31). Regarding the upper frequency specification, the average propagation constant is $\bar{\beta}_{u p}\left(f=f_{u p}\right)=210.76 \mathrm{rad} / \mathrm{m}$. The use of the average propagation constant implies that the synthesis process is going to be performed in the normalized propagation axis, $\chi$, and the real position $z$ will be determined in a subsequent step.

Moreover, as it was stated in Section IV, we need to calculate $\rho_{0}$ and $\rho_{0}^{\prime}$ for $\bar{\beta}_{\text {min }}=\bar{\beta}\left(f=f_{\text {min }}\right)$. In the particular case of a rectangular waveguide, $\rho_{0}$ and $\rho_{0}^{\prime}$ are directly expressed as a function of $f_{\text {min }}$ through (34) and (35). Solving both expressions for $f_{\min }=11 \mathrm{GHz}$, and the aforementioned waveguide dimensions at the taper extremes, we obtain $\rho_{0}=0.227$ and $\rho_{0}^{\prime}$ $=0.231$.
The coupling coefficient along the normalized propagation axis, $K(\chi)$, of the Hecken taper can be directly calculated by (18) using $\bar{\beta}_{\text {min }}$ and $\rho_{0}^{\prime}$ and it is represented in Fig.9. Then, due to the fact that the height is constant, $K_{b}(\chi)=0$ because of (36) and $k_{b}=0$ as well. Hence, $k_{a}=1$ leads to $K_{a}(\chi)=K(\chi)$ in (37) and the $\mathrm{a}(\chi)$ profile shown in Fig.9 can be calculated employing (41). Finally, in order to calculate the waveguide width along the actual position axis $z$, we must define the transformation frequency, $f_{t}$, at the center of the required matched frequency range, i.e., $f_{t}=12 \mathrm{GHz}$. Hence, $\bar{\beta}_{t}\left(f=f_{t}\right)=182.31 \mathrm{rad} / \mathrm{m}$. By employing $\bar{\beta}_{t}$ and $\beta_{t}\left(f_{t}, \chi\right)$ in (26), we can obtain the final width of the waveguide along the propagation direction, $a(z)$, that is also included in Fig.9. The length of the resulting Hecken taper is $L=30.04 \mathrm{~mm}$.

For the case of the novel multisection Chebyshev transformer-based taper, an output auxiliary impedance, $Z_{p 2}=$ $1.587 \Omega$, is calculated for $\rho_{0}=0.227$ and $Z_{p 1}=1 \Omega$ by means of (22). A minimum order $M=2$ and $\bar{\beta}_{0}=184.97 \mathrm{rad} / \mathrm{m}$, yielding to $\bar{\beta}_{\text {max }}=219.24 \mathrm{rad} / \mathrm{m}$ using (24), are determined for complying with the requirements as $\bar{\beta}_{\text {max }} \geq \bar{\beta}_{\text {up }}$. The characteristic impedances of the multisection Chebyshev transformer are $Z_{1}=1.128 \Omega$ and $Z_{2}=1.407 \Omega$. Once these

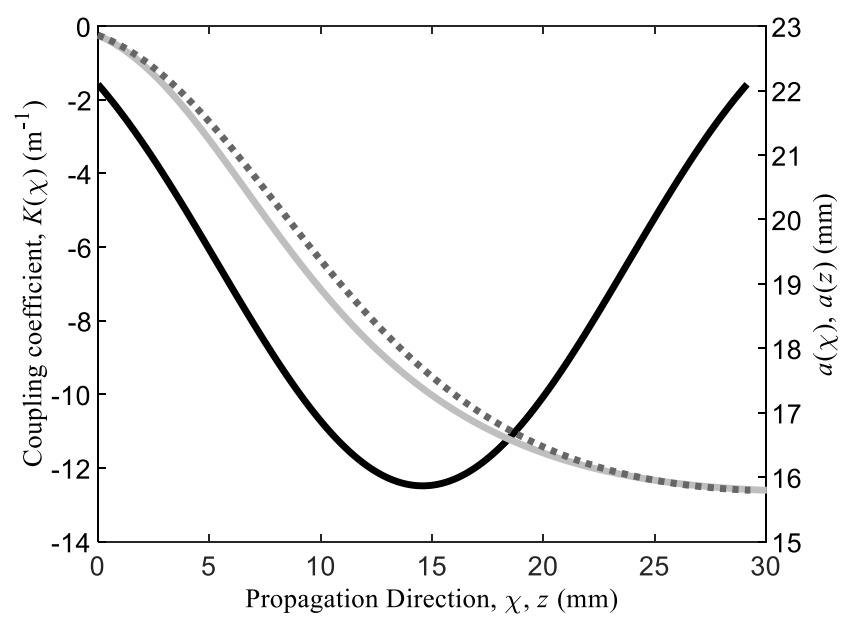

Fig. 9. Hecken taper: Coupling coefficient (black solid line) and width profile dimensions (dark grey dashed line) along the normalized propagation axis, $\chi$. The final width profile along the actual propagation axis, $z$, is also included (light grey solid line).

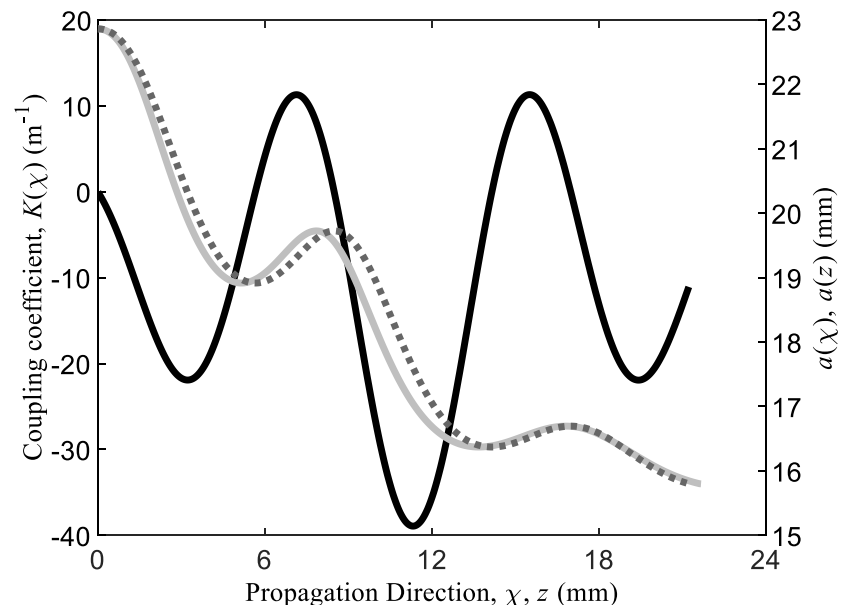

Fig. 10. Novel taper based on a 2-nd order multisection Chebyshev transformer: Coupling coefficient (black solid line) and width profile dimensions (dark grey dashed line) along the normalized propagation axis, $\chi$. The final width profile along the actual propagation axis, $z$, is also included (light grey solid line). 


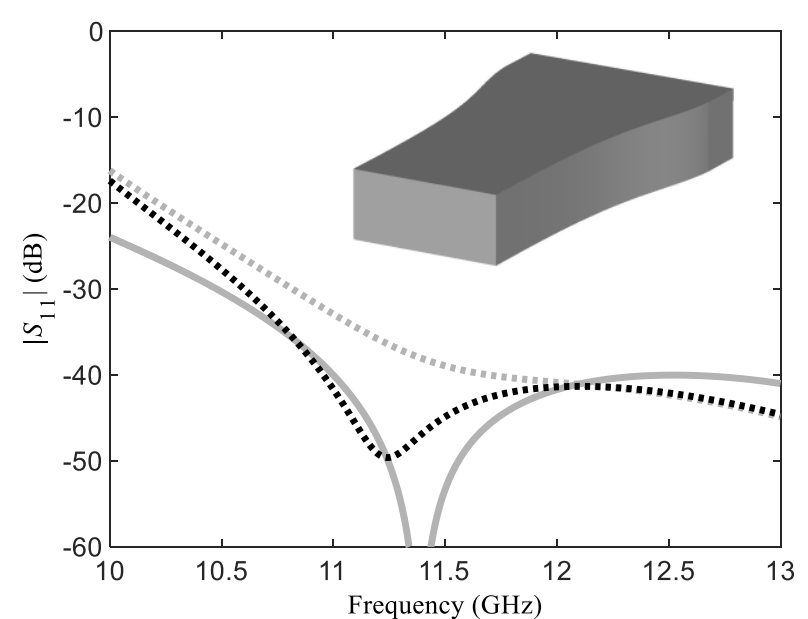

Fig. 11. Comparison of $\left|S_{11}(f)\right|$-parameters of the Hecken taper. Target response (grey solid line) and CST Microwave Studio simulations: taper with $a(\chi)$ width profile (grey dotted line) and taper with $a(z)$ width profile (black dotted line). Inset: view of the inner hollow volume of the definitive taper.

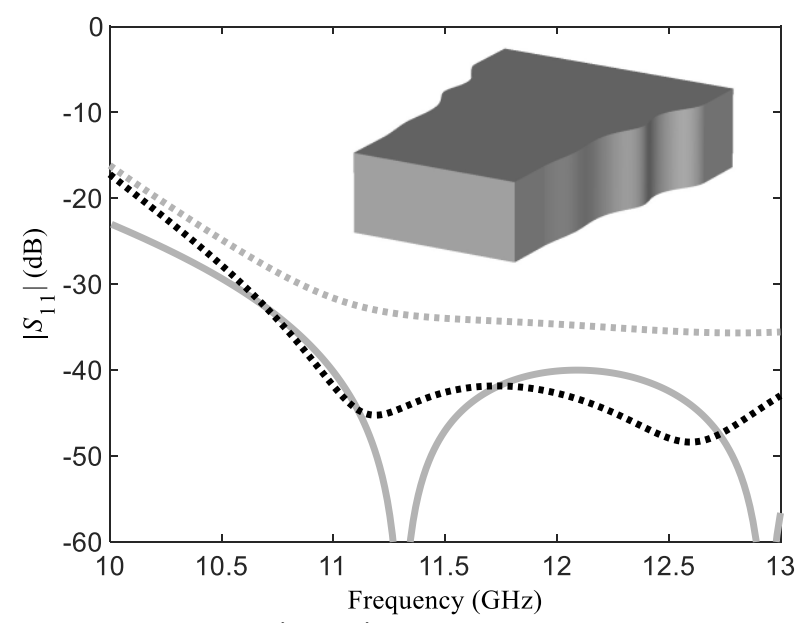

Fig. 12. Comparison of $\left|S_{11}(f)\right|$-parameters of the novel taper based on 2-nd order multisection Chebyshev transformer. Target response (grey solid line) and CST Microwave Studio simulations: taper with $a(\chi)$ width profile (grey dotted line) and taper with $a(z)$ width profile (black dotted line). Inset: view of the inner hollow volume of the definitive taper.

characteristic impedances are known, the $S_{11}(\bar{\beta})$ is calculated making use of the transmission matrix by defining $\bar{\beta}$ from 0 $\mathrm{rad} / \mathrm{m}$ to $50 \cdot \bar{\beta}_{0}$ every $\bar{\beta}_{0} / 50$. The final response intended to be synthetized is attained after setting $S_{11}\left(\bar{\beta}>\bar{\beta}_{z}\right)=0$ for $\bar{\beta}_{z}=$ $3 \cdot \bar{\beta}_{0}$. Then, $K(\chi)$ is calculated with (7) and the result is depicted in Fig.10. As $K_{b}(\chi)$ must be $0, k_{a}=1$ and $K_{a}(\chi)=K(\chi)$. The width dimensions of the rectangular waveguide taper along the normalized propagation axis, $\mathrm{a}(\chi)$, were calculated with (41) and are also displayed in Fig.10. The last transformation step is carried out like in the case of the Hecken taper case by means of (26) for $\bar{\beta}_{t}\left(f_{t}\right)$ with $f_{t}=12 \mathrm{GHz}$ and hence, the relation $z(\chi)$ is obtained. The resulting $a(z)$ profile is presented in Fig.10. The final Chebyshev transformer-base taper has a length $L=21.67$ $\mathrm{mm}$.

A CST Microwave Studio simulation is performed for the Hecken taper as well as for the one based on the 2-nd order multisection Chebyshev transformer, and the attained $\left|S_{11}(f)\right|$ parameters are shown in Fig.11 and Fig.12, respectively. An excellent agreement is achieved between both simulations and their corresponding target frequency responses. Moreover, the matching specifications are also fulfilled in terms of frequency range and level.

To conclude this subsection that shows the feasibility of the method to synthesize tapers for rectangular waveguides of different widths and same height, a final remark must be done. If we pay attention to the waveguide width profiles, $a(\chi)$ and $a(z)$, that have been obtained and shown in Fig. 9 for the Hecken taper as well as in Fig.10 for the novel taper, we can realize that $a(\chi)$ and $a(z)$ are pretty similar to each other in both cases. Therefore, one might think that the propagation axis transformation of (26) may not be actually necessary because the transformed width profile, $a(\chi)$, is close enough to the actual one, $a(z)$. As a result, it could be considered that if the taper of $a(z)$ meets the specifications, one defined by $a(\chi)$ perhaps meets them too, something that would make the propagation axis transformation in a trivial and useless synthesis step. Additional CST Microwave Studio simulations have been done with both tapers but defining the width profile with $a(\chi)$ instead of $a(z)$. The results of those simulations are displayed in Fig.11 for the Hecken taper and in Fig.12 for the Chebyshev transformer-based taper. In both cases, the obtained frequency response does not meet the required specifications and, actually, it is quite far from the expected behavior of the taper. This demonstrates the importance of the propagation axis transformation.

\section{Rectangular Waveguide Tapers Implemented with Variations in Height and Width Simultaneously}

Now that in previous subsections several examples have been presented of tapers matching rectangular waveguides where only the width or height changed, this subsection will be devoted to show how to synthetize tapers for the most complicated case where the cross-sections of the both waveguides do not have neither the same height nor same width. A Klopfenstein taper, a Hecken taper, and three different models of multisection Chebyshev transformer-based tapers will be synthetized for this case.

The specifications are going to be the same as with width variations only, i.e., a minimum $R L$ level of $40 \mathrm{~dB}\left(\rho_{m}=0.01\right)$ between $f_{\text {low }}=11 \mathrm{GHz}$ and $f_{\text {up }}=13 \mathrm{GHz}$. In order to obtain the shortest tapers, we impose $f_{\text {low }}=f_{\text {min }}$.

Regarding the geometry of the taper, the incoming waveguide would be WR90 whereas the outcoming waveguide is WR62. This means that $a(0)=22.86 \mathrm{~mm}, b(0)=10.16 \mathrm{~mm}, a(L)=$ $15.799 \mathrm{~mm}$, and $b(L)=7.899 \mathrm{~mm}$. Thus, $\rho_{0}=0.1046$ and $\rho_{0}^{\prime}=$ 0.105 are calculated by (34) and (35). The average propagation constant, calculated by $(31)$, for $f_{\min }$ is $\bar{\beta}_{\min }\left(f=f_{\min }\right)=$ $150.88 \mathrm{rad} / \mathrm{m}$, while for $f_{u p}$ is $\bar{\beta}_{u p}\left(f=f_{u p}\right)=210.76 \mathrm{rad} / \mathrm{m}$.

Both Klopfenstein and Hecken tapers are synthetized by (17) and (18) respectively using the calculated values of $\bar{\beta}_{\min }, \rho_{0}^{\prime}$ and $\rho_{m}$. The coupling coefficient along the normalized position is depicted in Fig. 13 for the Klopfenstein case whereas for the Hecken taper is shown in Fig.14.

For the synthesis of the three novel tapers, two different starting prototypes of multisection Chebyshev transformers have been considered, being $Z_{p 2}=1.234 \Omega$ the auxiliary characteristic impedance for $\rho_{0}$. The first transformer has an order $M=2$ with $\bar{\beta}_{0}=206.62 \mathrm{rad} / \mathrm{m}$, and hence $\bar{\beta}_{\max }=263.17$ $\mathrm{rad} / \mathrm{m}$, which is higher than $\bar{\beta}_{u p}$ guaranteeing that the specifications are met. The normalized characteristic impedances of the commensurate lines are $Z_{1}=1.059 \Omega$ and 
$Z_{2}=1.165 \Omega$. This allows us to calculate the $S_{11}(\bar{\beta})$ of the transformer that is going to be employed to synthetize two different responses: the first one is modified by applying $S_{11}\left(\bar{\beta}>\bar{\beta}_{z}\right)=0$ for $\bar{\beta}_{z}=3 \cdot \bar{\beta}_{0}$, while for the second one $\bar{\beta}_{z}=5 \cdot \bar{\beta}_{0}$ was chosen. The synthesis of those responses employing (7) results in the coupling coefficients that can be seen in the Fig.15 for $\bar{\beta}_{z}=3 \cdot \bar{\beta}_{0}$ and in Fig. 16 for $\bar{\beta}_{Z}=5 \cdot \bar{\beta}_{0}$. On the other hand, the second initial prototype is a 3 -rd order transformer centered at $\bar{\beta}_{0}=269.39 \mathrm{rad} / \mathrm{m}$, which extends its matched bandwidth from $\bar{\beta}_{\text {min }}$ up to $\bar{\beta}_{\text {max }}=388.7 \mathrm{rad} / \mathrm{m}$, with $Z_{1}=1.039 \Omega, Z_{2}=1.111$ $\Omega$, and $Z_{3}=1.188 \Omega$. The frequency response of this initial transformer is calculated and then modified with $\bar{\beta}_{z}=3 \cdot \bar{\beta}_{0}$ and synthetized by means of (7). The resulting coupling coefficient is shown in Fig.17.

Since all the tapers synthetized are intended to have the same cross-sections at their extremes, the constants calculated by (38) and (39) are the same for all of them: $k_{b}=-1.199$ and $k_{a}=2.199$. Then, $K_{b}(\chi)$ and $K_{a}(\chi)$ can be determined for each taper and this allows to obtain $b(\chi)$ and $a(\chi)$ afterwards by means of (40) and (41). Finally, the transformation to the real propagation axis, $z(\chi)$, is performed employing (26) for $\bar{\beta}_{t}\left(f=f_{t}\right)=182.31$ $\mathrm{rad} / \mathrm{m}$ with $f_{t}=12 \mathrm{GHz}$. The final width and height profiles of both Klopfenstein and Hecken tapers are shown in Fig.13 and Fig.14 respectively. The profiles corresponding to the novel tapers based on the $M=2$ Chebyshev transformer with $\bar{\beta}_{z}=3 \cdot \bar{\beta}_{0}$ and $\bar{\beta}_{z}=5 \cdot \bar{\beta}_{0}$ are depicted in Fig.15 and Fig.16. Finally, the taper based on the 3-rd order Chebyshev transformer with $\bar{\beta}_{z}=$ $3 \cdot \bar{\beta}_{0}$ is shown in Fig. 17.

The main parameters of all synthetized tapers as well as their final lengths are summarized in Table 1.

TABLE I

SYNTHESIS PARAMETERS FOR DIFFERENT WR-90 TO WR-62 TAPERS OPERATING FROM $11 \mathrm{GHZ}$ TO $13 \mathrm{GHZ}$ WITH RL $\geq 40$ DECIBELS AND THEIR LENGTHS

\begin{tabular}{lcccc}
\hline \hline \multicolumn{1}{c}{ Taper } & $M$ & $\bar{\beta}_{0}(\mathrm{rad} / \mathrm{m})$ & $\bar{\beta}_{Z}(\mathrm{rad} / \mathrm{m})$ & $L(\mathrm{~mm})$ \\
\hline Klopfenstein & - & - & & 20.65 \\
Hecken & - & - & & 23.93 \\
MCTB*1 & 2 & 206.62 & $3 \cdot \bar{\beta}_{0}$ & 19.37 \\
MCTB $^{*} 2$ & 2 & 206.62 & $5 \cdot \bar{\beta}_{0}$ & 17.89 \\
MCTB*3 & 3 & 269.39 & $3 \cdot \bar{\beta}_{0}$ & 20.82 \\
\hline * MCTB: Multisection Chebyshev Transformer-Based.
\end{tabular}

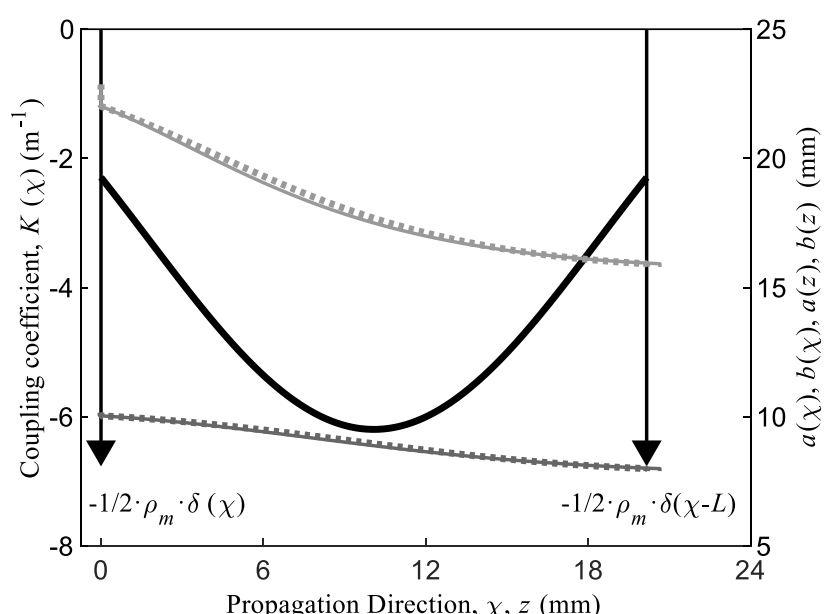

Fig. 13. Klopfenstein taper: Coupling coefficient (black solid line) as well as width (light grey dashed line) and height (dark grey dashed line) profile dimensions along the normalized propagation axis, $\chi$. The final width (light grey solid line) and height (dark grey solid line) profiles along the actual propagation axis, $z$, are also included.

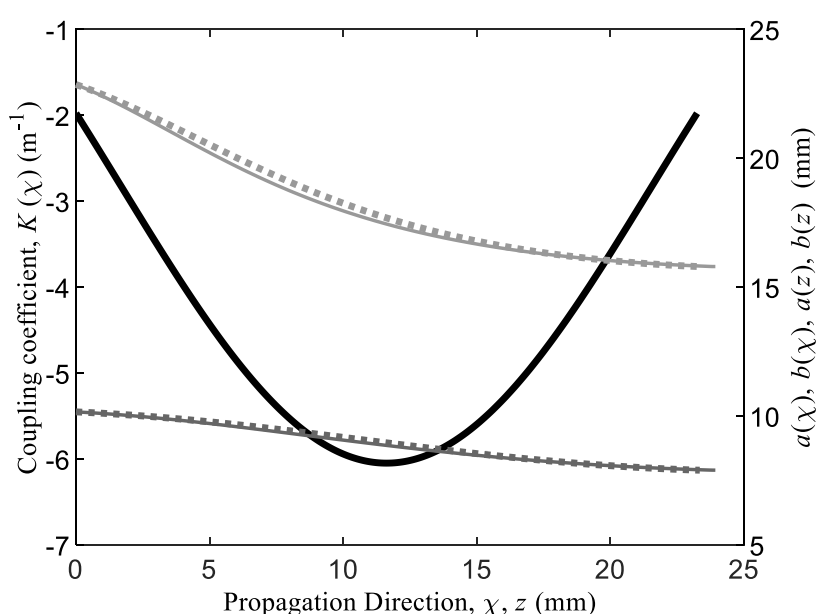

Fig. 14. Hecken taper: Coupling coefficient (black solid line) as well as width (light grey dashed line) and height (dark grey dashed line) profile dimensions along the normalized propagation axis, $\chi$. The final width (light grey solid line) and height (dark grey solid line) profiles along the actual propagation axis, $z$, are also included.

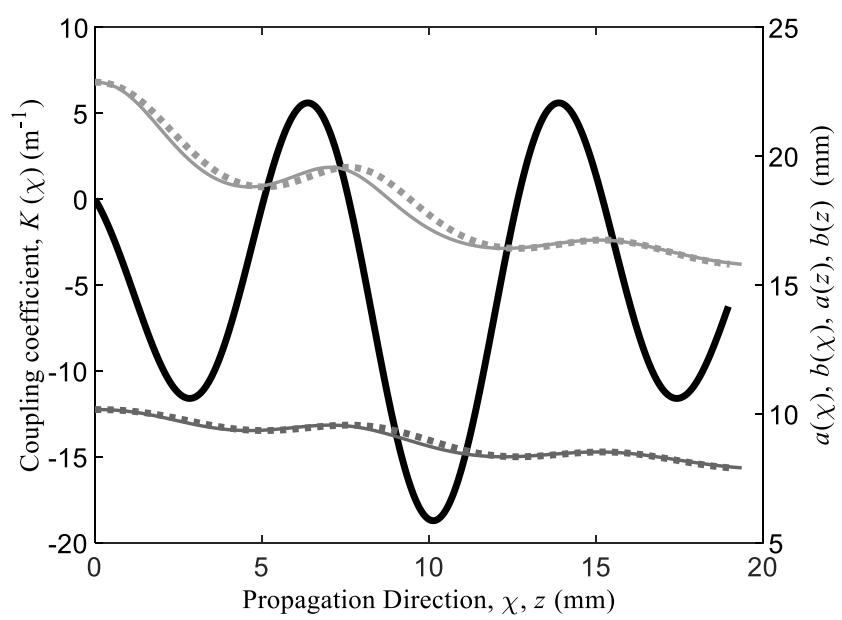

Fig. 15. Novel taper based on a 2-nd order multisection Chebyshev transformer with $\beta_{z}=3 \cdot \beta_{0}$ : Coupling coefficient (black solid line) as well as width (light grey dashed line) and height (dark grey dashed line) profile dimensions along the normalized propagation axis, $\chi$. The final width (light grey solid line) and height (dark grey solid line) profiles along the actual propagation axis, $z$, are also included.

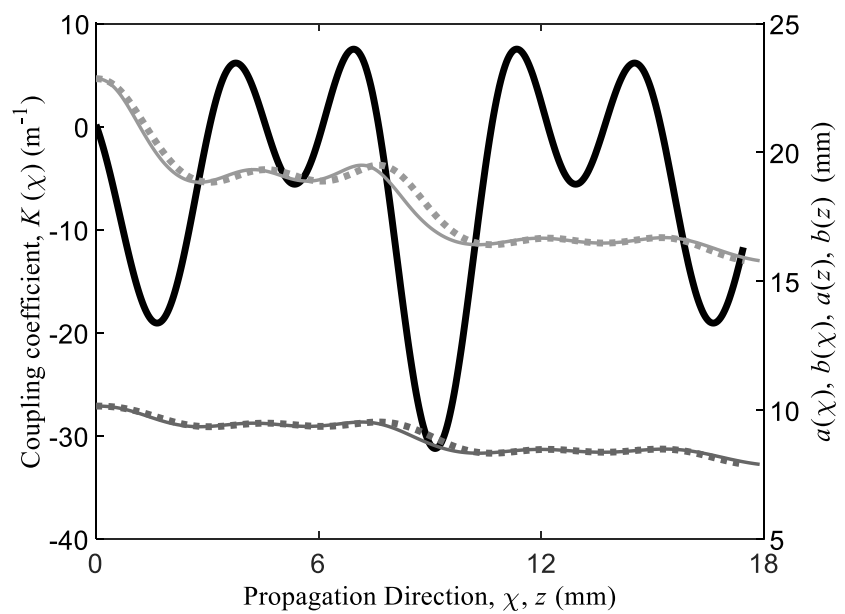

Fig. 16. Novel taper based on a 2-nd order multisection Chebyshev transformer with $\beta_{z}=5 \cdot \beta_{0}$ : Coupling coefficient (black solid line) as well as width (light grey dashed line) and height (dark grey dashed line) profile dimensions along the normalized propagation axis, $\chi$. The final width (light grey solid line) and height (dark grey solid line) profiles along the actual propagation axis, $z$, are also included. 


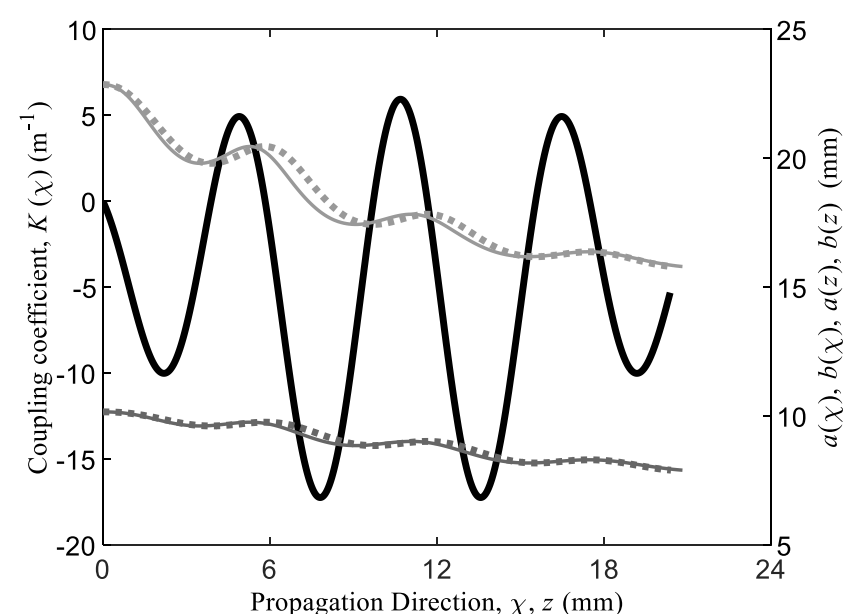

Fig. 17. Novel taper based on a 3-rd order multisection Chebyshev transformer with $\beta_{z}=3 \cdot \beta_{0}$ : Coupling coefficient (black solid line) as well as width (light grey dashed line), and height (dark grey dashed line) profile dimensions along the normalized propagation axis, $\chi$. The final width (light grey solid line) and height (dark grey solid line) profiles along the actual propagation axis, $z$, are also included.

The five different tapers synthetized with height and width variations have been fabricated in AlSi10Mg aluminum alloy, by means of an Additive Manufacturing technique (Direct Metal Laser Sintering), using an EOS M290 printer which allows a maximum building volume of $250 \times 250 \times 350 \mathrm{~mm}$. All the tapers were fabricated growing the structures from the WR62 to the WR90 port, following the propagation direction in order to avoid overhanging surfaces. A photograph of the prototypes is shown in Fig. 18. The DMLS technique was selected since it has been lately employed to fabricate inexpensive RF parts from a few $\mathrm{GHz}$ to $30 \mathrm{GHz}$, approximately [41]. Beyond that frequency, the fabrication accuracy of DMLS begins to become insufficient. Nevertheless, if specifications for higher frequencies are required, metal coated polymer Additive Manufacturing solutions like Stereolithography Apparatus (SLA) or Fused Deposition Modeling (FDM), among others, could be employed, since they achieve significantly better accuracies. Alternatively, the classical electroforming manufacturing technique could be also used for an inexpensive fabrication of these structures, for example.

Since the designed tapers operate between different waveguide standards, two different measurement set-ups had to be employed to test the tapers using a Keysight (Agilent) 8722 Vector Network Analyzer (VNA). The first one (set-up 1) consisted in calibrating the VNA with a WR90 calibration kit and the tapers were tested loading their WR62 port with a waveguide sliding load of a WR62 calibration kit. For the second set-up (set-up 2) the VNA was calibrated using a WR62 calibration kit and the tapers were tested loading the WR90 port with the sliding load of a WR90 calibration kit.

A comparison between the target responses, CST Microwave Studio simulations and measurement results is shown for each taper in Fig. 19 to Fig.23.
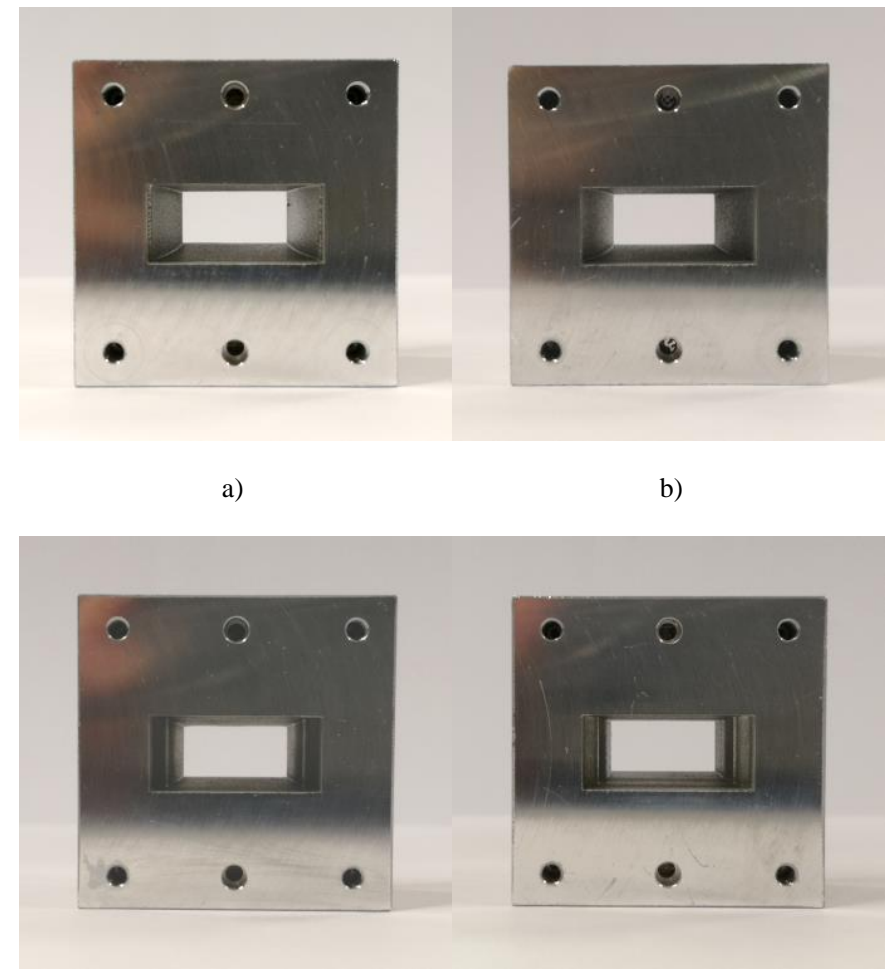

c)

d)

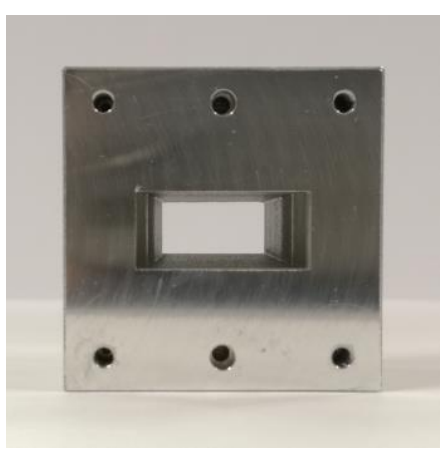

e)

Fig. 18. Photographs of the tapers fabricated by means of Direct Metal Laser Sintering technique: a) Klopfenstein taper; b) Hecken taper; multisection Chebyshev transformer-based taper with c) $M=2, \bar{\beta}_{z}=3 \cdot \bar{\beta}_{0}$; d) $M=2, \bar{\beta}_{z}=5 \cdot \bar{\beta}_{0}$; and e) $M=3, \bar{\beta}_{z}=3 \cdot \bar{\beta}_{0}$.

A very good agreement is achieved between simulation and measurement results in all cases. In fact, the aim specifications, $R L \geq 40 \mathrm{~dB}$ from $f_{\text {low }}=11 \mathrm{GHz}$ up to $f_{\text {up }}=13 \mathrm{GHz}$, are fully achieved in simulation and very close to be achieved in measurements. The small discrepancies found between simulations and measurements can be attributed to two main reasons. The first one is related to the DMLS manufacturing tolerances, as fabrication inaccuracies of $\pm 100 \mu \mathrm{m}$ can be easily expected using this technique of Additive Manufacturing of metallic parts. However, the main reason for discrepancies is the measurement set-up itself, because two sliding waveguide loads were employed to load the port that was not connected to the VNA. During the measurements, these loads were not operating in their native frequency range since the VNA is calibrated for another standard with its own optimal frequency range. Actually, measurements made with set-up 1 are more troublesome than measurements performed with set-up 2, because the WR62 load is operating from $10 \mathrm{GHz}$ to $13 \mathrm{GHz}$, and the WR62 standard range starts at $12.4 \mathrm{GHz}$. Nevertheless, 
the propagation regime is ensured for WR62 in the whole operation bandwidth. On the other hand, set-up 2 employs the WR90 load from $10 \mathrm{GHz}$ to $13 \mathrm{GHz}$, and the native WR90 bandwidth is defined from $8.2 \mathrm{GHz}$ to $12.4 \mathrm{GHz}$. Finally, it must be pointed out that a minor shift to lower frequencies can be observed when comparing the target and the simulated frequency responses. That frequency shift is caused by parasitic reactive coupling to higher order modes that are under cut-off, but suffer a small excitation somewhat noticeable for these tapers with simultaneous variations in height and width. However, since the minor shift is towards lower frequencies, the tapers obtained continue fulfilling the aim specifications as it has been shown in the simulation and measurement results.

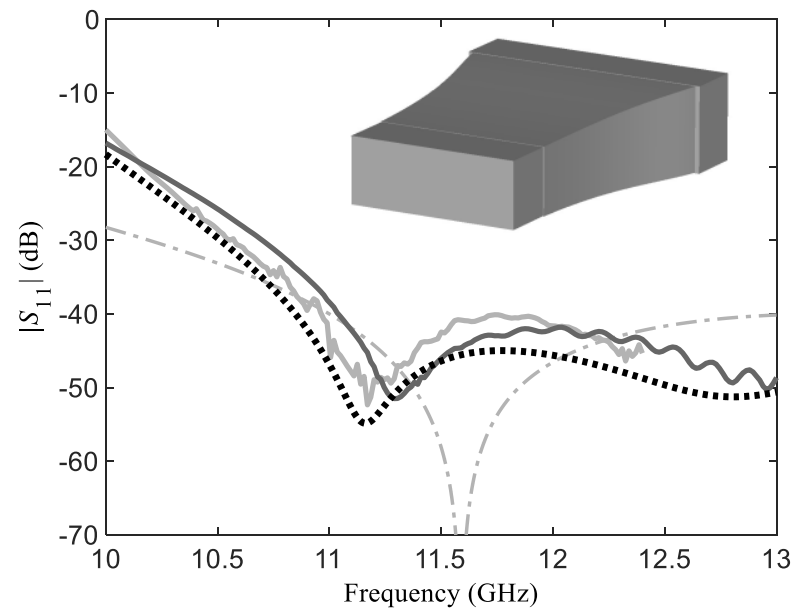

Fig. 19. Comparison of $\left|S_{11}(f)\right|$-parameters of the Klopfenstein taper: target response (grey dash-dotted line), CST Microwave Studio simulation (black dotted line), measurements performed with WR90 (light grey solid line) and WR62 (dark grey solid line) calibration. Inset: view of the inner hollow volume of the taper.

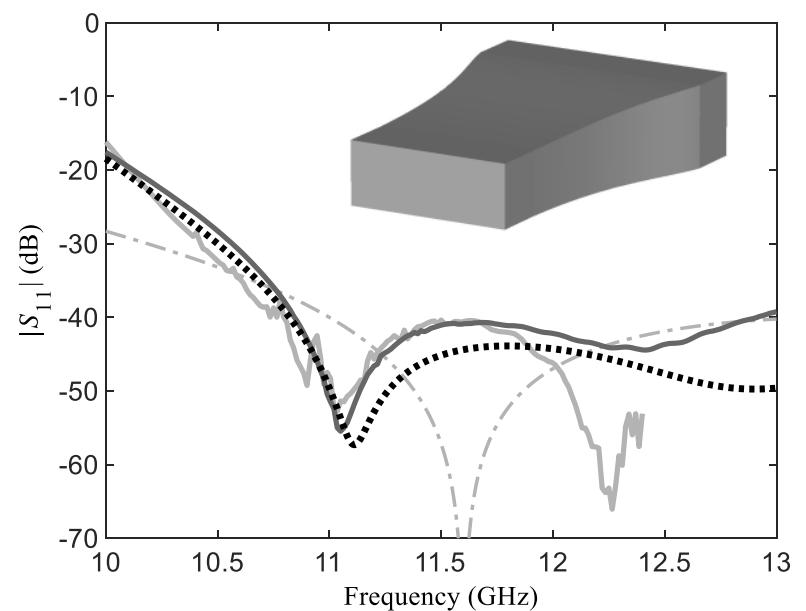

Fig. 20. Comparison of $\left|S_{11}(f)\right|$-parameters of the Hecken taper: target response (grey dash-dotted line), CST Microwave Studio simulation (black dotted line), measurements performed with WR90 (light grey solid line) and WR62 (dark grey solid line) calibration. Inset: view of the inner hollow volume of the taper.

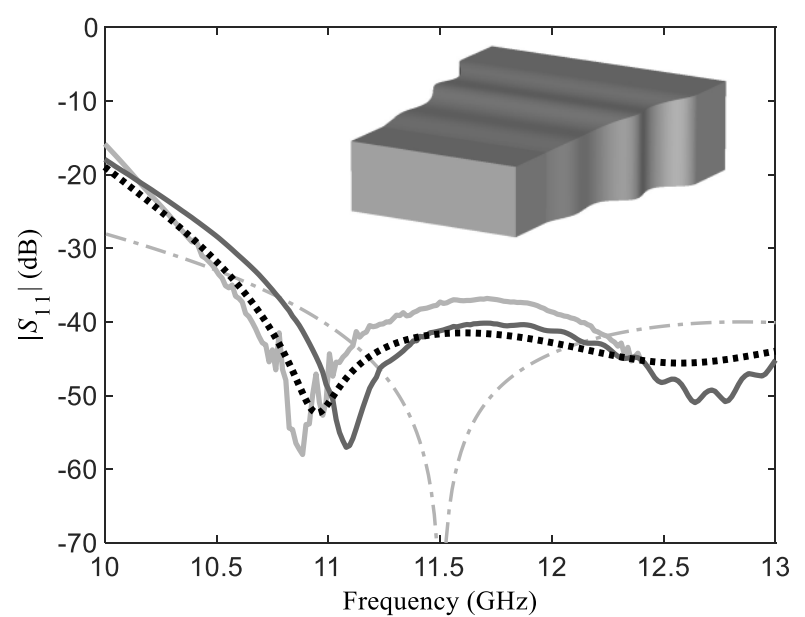

Fig. 21. Comparison of $\left|S_{11}(f)\right|$-parameters of the novel taper based on 2-nd order multisection Chebyshev transformer with $\beta_{z}=3 \cdot \beta_{0}$ : target response (grey dash-dotted line), CST Microwave Studio simulation (black dotted line), measurements performed with WR90 (light grey solid line) and WR62 (dark grey solid line) calibration. Inset: view of the inner hollow volume of the taper.

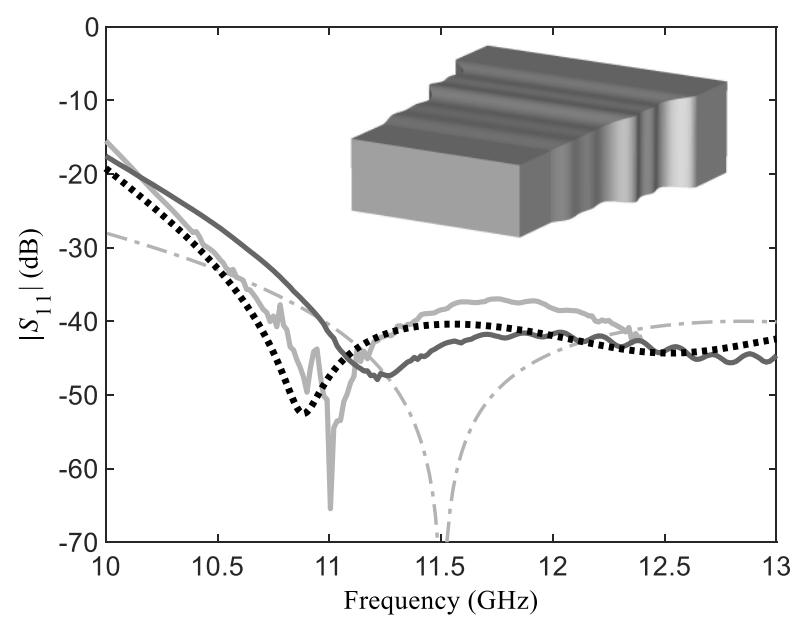

Fig. 22. Comparison of $\left|S_{11}(f)\right|$-parameters of the novel taper based on 2 -nd order multisection Chebyshev transformer with $\beta_{z}=5 \cdot \beta_{0}$ : target response (grey dash-dotted line), CST Microwave Studio simulation (black dotted line), measurements performed with WR90 (light grey solid line) and WR62 (dark grey solid line) calibration. Inset: view of the inner hollow volume of the taper.

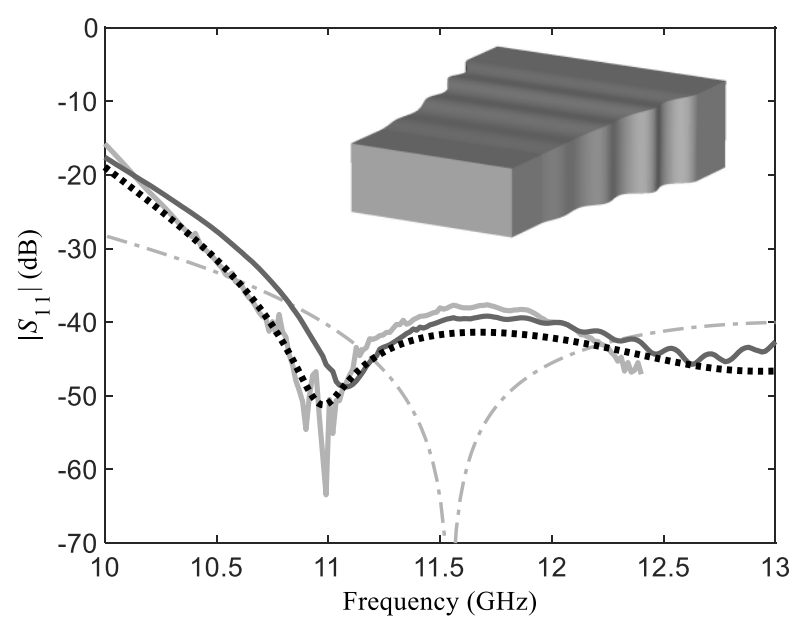

Fig. 23. Comparison of $\left|S_{11}(f)\right|$-parameters of the novel taper based on 3-rd order multisection Chebyshev transformer with $\beta_{z}=3 \cdot \beta_{0}$ : target response (grey dash-dotted line), CST Microwave Studio simulation (black dotted line), measurements performed with WR90 (light grey solid line) and WR62 (dark grey solid line) calibration. Inset: view of the inner hollow volume of the taper. 


\section{CONCLUSION}

A novel synthesis technique for waveguide tapers intended to operate over a frequency range with single mode propagation has been carefully explained and demonstrated. The technique employs the coupled-mode theory in order to characterize the electromagnetic behavior of the synthesized tapered matching section. By using a series solution of the single mode synthesis problem, and its 0 -th order low reflectivity approximation, analytical expressions for the coupling coefficient of the classical Klopfenstein and Hecken tapers are obtained. From the coupling coefficient, the physical waveguide dimensions can be calculated, allowing us to extend the classical transmission line taper solutions to general waveguide tapers with single mode operation. Additionally, a new family of tapers has been proposed, obtained by applying the series solution of the synthesis problem to properly modified frequency responses of multisection Chebyshev transformers. This procedure gives rise to smooth waveguide tapers that can be even shorter than the Klopfenstein taper.

The synthesis technique has been validated through several examples in rectangular waveguide technology. The tapers required the matching of waveguide cross-sections of different heights and/or widths. Both classical and new taper functions have been demonstrated. Some of these tapers were fabricated by means of an Additive Manufacturing technique for metallic parts. Simulation and measurement results confirm the high reliability and excellent performance the novel taper synthesis method reported.

\section{REFERENCES}

[1] E. Dietze, "Tapered Filter for Alternating Currents of Varying Frequency," U. S. Patent 1,603,329; Oct. 1926.

[2] H. A. Wheeler, "Transmission Lines with Exponential Taper," Proc. IRE, vol. 27, no. 1, pp. 65-71, Jan. 1939.

[3] A. W. Gent and P. J. Wallis, "Impedance Matching by Tapered Transmission Lines," J. Inst. Electr. Eng. 3, vol. 93, no. 3, pp. 559-563, 1946.

[4] D. M. Pozar, Microwave Engineering, $4^{\text {th }}$ ed. Norwood, NJ, USA: Wiley, 2005.

[5] R. E. Collin, Foundations for Microwave Engineering, Second Edition, New York, McGraw Hill, 1992.

[6] R. W. Klopfenstein, "A Transmission Line Taper of Improved Design," Proc. IRE, vol. 44, pp. 31-35, Jan. 1956.

[7] R. E. Collin, "The Optimum Tapered Transmission Line Matching Section," Proc. IRE, vol. 44, no. 4, pp. 539-548, April 1956.

[8] J. A. Fuchs, "Tapered Transmission Line Computer Program Using Collin's Derivation," Proc. IEEE, vol. 56, no. 12, pp. 2174-2175, Dec. 1968.

[9] D. Kajfez and J. O. Prewitt, "Correction to "A Transmission Line Taper of Improved Design" (Letters)," IEEE Trans. Microw. Theory Techn., vol. 21, no. 5, pp. 364-364, May 1973.

[10] R. P. Hecken, "A Near Optimum Matching Section without Discontinuities, “IEEE Trans. Microw. Theory Techn., vol. MTT-20, pp. 734-739, Nov. 1972.

[11] P. Pramanick and P. Bhartia, "A Generalized Theory of Tapered Transmission Tine Matching Transformers and Asymmetric Couplers Supporting non-TEM Modes," IEEE Trans. Microw. Theory Techn., vol. 37, no. 8, pp. 1184-1191, Aug. 1989

[12] M. Kobayashi and N. Sawada, "Analysis and Synthesis of Tapered Microstrip Transmission Lines," IEEE Trans. Microw. Theory Techn., vol. 40, no. 8, pp. 1642-1646, Aug. 1992.

[13] J. H. Hinken, "Simplified Analysis and Synthesis of Fin-line Tapers," Arch. Elek. Ubertrugung., vol. 37, pp. 375-380, Nov.-Dec. 1983.

[14] C. Schieblich, J. K. Piotrowski, and J. H. Hinken, "Synthesis of Optimum Finline Tapers Using Dispersion Formulas for Arbitrary Slot Widths and Locations," IEEE Trans. Microw. Theory Techn., vol. 32, no. 12, pp. 16381645, Dec. 1984.

[15] F. Sporleder, and H. G. Unger, Waveguide Tapers, Transitions and Couplers, London, UK: Peter Peregrinus Ltd., 1979.
[16] B. Z. Katsenelenbaum et al., Theory of Nonuniform Waveguides-the Cross-Section Method, London, UK: IEE Electromagnetic Waves Series, 44, 1998.

[17] L. Solymar, "Design of a Conical Taper in Circular Waveguide System Supporting $\mathrm{H}_{10}$ Mode," Proc. IRE, vol. 46, no. 3, pp. 618-619, March 1958.

[18] R. C. Johnson, "Design of Linear Double Tapers in Rectangular Waveguides," IRE Trans. Microw. Theory Techn., vol. 7, no. 3, pp. 374378, July 1959.

[19] K. Matsumaru, "Reflection of a Pyramidally Tapered Rectangular Waveguide," IRE Trans. Microw. Theory Techn., vol. 7, no. 2, pp. 192196, April 1959.

[20] L. Solymar, "Design of a Two-section Conical Taper in Circular Waveguide System Supporting the $\mathrm{H}_{01}$ mode," Proc. IEE - Part B: Electronic and Communication Engineering, vol. 106, no. 13, pp. 119-120, Jan. 1959.

[21] L. Solymar, "Monotonic Multi-section Tapers for Over-moded Circular Waveguides," Proc. IEE - Part B: Electronic and Communication Engineering, vol. 106, no. 13, pp. 121-128, Jan. 1959.

[22] H. G. Unger, "Circular Waveguide Taper of Improved Design," Bell Syst. Tech. J., vol. 37, pp. 899-912, July 1958.

[23] C. C. H. Tang, "Optimization of Waveguide Tapers Capable of Multimode Propagation," IRE Trans. Microw. Theory Techn., vol. 9, no. 5, pp. 442452, Sept. 1961.

[24] R. P. Hecken and A. Anuff, "On the Optimum Design of Tapered Waveguide Transitions," IEEE Trans. Microw. Theory Techn., vol. 21, no. 6, pp. 374-380, June 1973.

[25] J. L. Doane, "Parabolic Tapers for Overmoded Waveguides," Int. J. Infrared Millim. Waves, vol. 5, no. 5, pp. 737-751, May 1984.

[26] F. Sporleder, "Waveguide Transition Design of Improved Accuracy," Arch. Elek. Ubertrugung., vol. 30, pp. 289-296, 1976

[27] H. Flugel and E. Kuhn, "Computer-aided Analysis and Design of Circular Waveguide Tapers," IEEE Trans. Microw. Theory Techn., vol. 36, no. 2, pp. 332-336, Feb. 1988.

[28] S. Cogollos et al., "Novel Planar and Waveguide Implementations of Impedance Matching Networks Based on Tapered Lines Using Generalized Superellipses," IEEE Trans. Microw. Theory Techn., vol. 66, no. 4, pp. 1874-1884, April 2018.

[29] I. Arnedo et al., "Synthesis of Microwave Filters by Inverse Scattering Using a Closed-Form Expression Valid for Rational Frequency Responses", IEEE Trans. Microw. Theory Techn., vol. 60, no. 5, pp. 12441257, May 2012

[30] I. Arnedo et al., "Direct and Exact Synthesis: Controlling the Microwaves by Means of Synthesized Passive Components with Smooth Profiles," IEEE Microw. Mag., vol. 16, no. 4, pp. 114-128, May 2015.

[31] I. Arnedo et al., "A Series Solution for the Single-Mode Synthesis Problem Based on the Coupled-Mode Theory," IEEE Trans. Microw. Theory Techn., vol. 56, no. 2, pp. 457-466, Feb. 2008.

[32] M. Chudzik et al., "Novel Synthesis Technique for Microwave Circuits Based on Inverse Scattering: Efficient Algorithm Implementation and Application", Int. J. RF Microw. C. E., vol. 21, no. 2, pp. 164-173, Mar. 2011.

[33] I. Arnedo et al., "Passive Microwave Planar Circuits for Arbitrary UWB Pulse Shaping," IEEE Microw. Compon. Lett., vol. 18, no. 7, pp. 452-454, July 2008.

[34] I. Arnedo et al., "Customized Pulses for UWB Applications," The 40th European Microwave Conf. (EuMC), Paris, 2010, pp. 320-323.M.

[35] Chudzik et al., "Design of Transmission-type Nth-order Differentiators in Planar Microwave Technology," IEEE Trans. Microw. Theory Techn., vol. 60, no. 11, Nov. 2012.

[36] J. M. Percaz et al., "Synthesis of Tapers Using the Coupled-Mode Theory," 2018 IEEE MTT-S Latin America Microwave Conf. (LAMC), Arequipa, 2018, pp. 1-4.

[37] A. Papoulis, The Fourier Integral and its applications. New York. McGraw-Hill Electronic Science Series, 1962.

[38] R. E. Collin, "Theory and Design of Wide-Band Multisection QuarterWave Transformers," Proc. IRE, vol. 43, no. 2, pp. 179-185, Feb. 1955.

[39] R. J. Cameron, C. M. Kudsia, and R. R. Mansour, Microwave Filters for Communication Systems: Fundamentals, Design and Applications. Hoboken, NJ, USA: Wiley, 2007.

[40] I. Arnedo et al., "Synthesis of One Dimensional Electromagnetic Bandgap Structures with Fully Controlled Parameters," IEEE Trans. Microw. Theory Techn., vol. 65, no. 9, pp. 3123-3134, Sept. 2017.

[41] R. Sorrentino, and O. A. Peverini, "Additive Manufacturing: a Key Enabling Technology for Next-Generation Microwave and MillimeterWave Systems," Proc. IEEE, vol. 104, no. 7, pp. 1362-1366, July 2016. 